\title{
Wind Turbine Asymmetrical Load Reduction with Pitch Sensor Fault Compensation $\|^{\dagger}$
}

\author{
Yanhua Liu | Ron J. Patton* Shuo Shi
}

\author{
${ }^{1}$ Department of Engineering, The \\ University of Hull, Cottingham Road, \\ Hull, HU6 7RX, UK \\ Correspondence \\ *Email: r.j.patton@hull.ac.uk
}

\begin{abstract}
Offshore wind turbines suffer from asymmetrical loading (blades, tower etc.), leading to enhanced structural fatigue. As well as asymmetrical loading different faults (pitch system faults etc.) can occur simultaneously, causing degradation of load mitigation performance. Individual pitch control (IPC) can achieve rotor asymmetric loads mitigation, but this is accompanied by an enhancement of pitch movements leading to the increased possibility of pitch system faults, which exerts negative effects on the IPC performance. The combined effects of asymmetrical blade and tower bending together with pitch sensor faults are considered as a "co-design" problem to minimize performance deterioration and enhance wind turbine sustainability. The essential concept is to attempt to account for all the "fault effects" in the rotor and tower systems which can weaken the load reduction performance through IPC. Pitch sensor faults are compensated by the proposed fault-tolerant control (FTC) strategy to attenuate the fault effects acting in the control system. The work thus constitutes a combination of IPC-based load mitigation and FTC acting at the pitch system level. An LQR-based IPC strategy for simultaneous blade \& tower loading mitigation is proposed in which the robust fault estimation is achieved using an unknown input observer (UIO), considering four different pitch sensor faults. The analysis of the combined UIO-based FTC scheme with the LQR-based IPC is shown to verify the robustness and effectiveness of these two systems acting together as well as separately.
\end{abstract}

\section{KEYWORDS:}

Fault-tolerant control, individual pitch control, pitch sensor faults, wind turbine asymmetrical load reduction

\section{1 | INTRODUCTION}

As a sustainable energy source, wind energy is taking an increasing share of the energy market to meet the growing demand for marine renewable energy. Wind energy has a significant potential for overcoming environmental pollution-related problems by reducing dependence on declining fossil fuel reserves. Wind turbines (WTs) can operate on land or offshore. Offshore WTs have large rotor diameters and high towers for high energy capture. The significant development of offshore wind power in recent years has led to a significant decrease in the levelized cost of energy (LCoE) of this form of renewable energy. However, there are two major challenges that the offshore WTs industry must face for Region 3 operation (above rated wind speed):

\footnotetext{
${ }^{\dagger}$ This is the peer reviewed version of the following article: [Yanhua Liu, Ron Patton and Shuo Shi. "Wind Turbine Asymmetrical Load Reduction with Pitch Sensor Fault Compensation". Wind Energy. 2020. Accepted.], which has been published in final form at [DOI:10.1002/we.2496]. This article is accepted on 17th Feb 2020 and published online on 11th Mar 2020. This article may be used for non-commercial purposes in accordance with Wiley Terms and Conditions for Use of Self-Archived Versions.
} 
(1) Unexpected malfunction and failures of WT components will result in expensive repairs and typically months of machine unavailability, thus increasing the operation and maintenance (O\&M) costs and threatening to increase the LCoE. However, offshore WT operation and maintenance are also challenged by the fact that windfarms are sometimes located $100 \mathrm{kms}$ offshore.

(2) The use of large rotors and towers with composite structures has led to a very significant increase in unbalanced or asymmetrical loading due to complex air flow, wind shear, gravity, yaw misalignment, tower shadow 1 , etc. The structures bend very significantly particularly at high wind velocity.

Therefore, it is of fundamental importance to design a sustainable control strategy with online fault diagnosis and fault-tolerant control. Robust knowledge of fault data will enable predictive maintenance schedules to be determined optimally and on-line fault tolerance will help to reduce the asymmetrical turbine rotor \& tower loading and avoid further unnecessary fault development. Moreover, the maintenance cost for tower and foundations contributes up to $12 \%$ of the cost of energy due to the harsh sea conditions ${ }^{2}$.

To alleviate the asymmetrical or unbalanced loads it is well known that individual pitch control (IPC) can mitigate the blade fatigue effectively 1 , 3 . This is achieved as in IPC the pitch angles are chosen using separate control signals to evoke a "dissimilar" redundant actuation system, as required to seek to balance the rotor. A common approach for achieving tower load alleviation is to add an extra collective blade pitch angle (collective pitch control, CPC) at the tower natural frequency based on the tower acceleration measurements 3 or estimated tower acceleration knowledge ${ }^{4}$. The authors ${ }^{4}$ propose a novel individual pitch-based tower damping control strategy based on estimated tower motion from blade load sensors. The benefit of this approach is that tower motion is controlled from the pitch tilt signal, thus achieving the decoupling with the power regulation loop. This method adopts the existing architecture of separate IPC, CPC and tower motion controllers. Typically, the currently understood load mitigation schemes are handled using separate control loops to avoid the couplings between each other.

However, it is found that because of the couplings between the blade flap and tower modes there exist some side effects from different load mitigation controllers when they perform separately. As shown in the work ${ }^{5}$, the use of a simple feedback signal proportional to the tower acceleration can be counter-productive causing an increase in tower fatigue loading rather than a reduction. Although the goal of the active tower damping controller is to reduce the tower fluctuations this fails to provide an effective impact on the blade loading alleviation, and it actually enhances the unbalanced blade loading in some cases 6 , 7. The loading on the rotor blades and yaw bearings are reduced by suitable IPC system design, without providing an effective mitigation of tower vibration. The latter may even be enhanced, as reported in 7 . Therefore, it is appealing to integrate the tower damping and blade load reduction (even reducing power output fluctuation) into one advanced multi-input multi-output (MIMO) control system. A good way to start this study is to use the LQR design as it is a relatively well-known method of achieving feedback control design. The value of LQR lies in the way in which the quadratic performance criterion is used to minimize the multivariable interactions (i) between the state variables and (ii) between the control signals. Estimates of pitch system faults are combined within the LQR-based IPC system to compensate for the fault action and enhance the actuation performance. Hence, the idea is to firstly demonstrate what can be achieved using combined LQR-based IPC and pitch fault estimation prior to extending the strategy to include more advanced control design, based on e.g. model predictive control (MPC), or the use of $\mathrm{H}_{\infty}$ synthesis design. For real system implementation MPC is often accompanied by relatively high computing loads and would require wind prediction information ${ }^{8}$. The latter is currently hard to achieve without using LiDAR or predicted wind knowledge. The advantage of LQR is that it can be used to apply state variable and control constraints through the quadratic performance index. On the other hand, $\mathrm{H}_{\infty}$ synthesis can be used and provides some advantage of dealing with robustness but does not facilitate the handling of state and control interactions ${ }^{9}$. As a starting point LQR provides a useful way of demonstrating the applicability of pitch system fault tolerant control (FTC) as a relatively simple and practicable combined LQR control and fault estimation scheme.

However, this load reduction performance by IPC comes with the sacrifice of enhanced pitch movements, which increases the pitch system fatigue and thus increases the likelihood of pitch system faults. The resulting pitch system faults will in turn deteriorate the IPC load reduction performance. Hence, it is important to jointly optimise the requirement for structural loading mitigation whilst restricting control demands for large pitch variations. Two ways to reduce the negative impacts of pitch faults are known in the literature:

(1) Decrease both the pitch travel and pitch rate caused by the additional pitch angle from the proposed IPC system 7, 10, this is to reduce the possibility of pitch faults developing.

(2) Enhance the robustness and reliability of the pitch control using fault estimation (FE) based FTC to compensate the undesirable effect that pitch faults have on the IPC system.

In line with the first approach, a more advanced load control strategy namely "smart rotor control" is proposed to maintain the load reduction performance without enhancing pitch movements using in-blade smart actuators 7 . However, this strategy requires an expensive blade redesign to obtain inputs from the built-in intelligent aerodynamic actuators located directly in the blades. This is not realistic for the existing WTs. Hence, the goal of this paper is to follow strategy (2) above using FE-based FTC as a mechanism to reduce the effect of these faults within the IPC system, and thereby sustaining the load balancing performance in a fault tolerant system.

Furthermore, the scenario of asymmetrical load mitigation is analogous to the FTC problem because the action of rotor bending (caused by wind loading) can itself be considered as a fault effect. A fault acting in a system is an unwanted effect causing a deterioration in performance and this 
is precisely what happens with rotor blade \& tower bending. So, it is quite attractive to consider all the "fault effects" acting in both the rotor and tower system, i.e. sensor faults, actuator faults and bending moment effects. The bending moment changes are effectively component faults acting in the rotor/tower system. The various objectives arising in the FTC and unbalanced load mitigation problems can then be viewed as a problem of "co-design", in which these features are handled by a joint form of multi-objective design.

Several research papers are proposed to deal with the wind turbine pitch sensor faults using model-based fault detection or FTC methods 11, 12, 13, 14, 15, 16, 17. The research 11 proposes a Kalman filter-based fault detection scheme using LMI-based residual evaluation for blade pitch actuator and sensor fault scenarios. The work ${ }^{12}$ proposes a model-based fault detection and diagnosis system using a data-driven fuzzy modeling and identification method considering the pitch actuator loss of effectiveness fault, which is verified in the IPC system. The work 13 proposes an FDI (fault detection, isolation)-based FTC strategy of a blade pitch system for various faults including pitch sensor biases, fixed outputs and stuck pitch actuators. However, these above strategies $11,12,13$ fail to provide useful information about the fault magnitude and suffer from the time delay which results from the use of residual-based fault diagnosis strategy. In the work ${ }^{14}$, the pitch sensor faults are estimated by an extended state observer while this paper $\frac{15}{15}$ presents an adaptive observer for pitch sensor fixed fault estimation in the presence of disturbance. It is clear that the fault estimation signals from the above two strategies suffer from considerable errors. A robust fault reconstruction strategy for pitch system faults using a modified sliding mode observer is shown to have good fault estimation accuracy in the work 16 . The model used for the above studies is the WT benchmark mode $[18$, which does not include the aero-elastic codes used in the NREL FAST WT simulator 19 and does not therefore have the required rotor system environment needed for IPC validation. A fault-tolerant tracking control system with a sliding mode observer for simultaneous WT pitch actuator/sensor fault estimation and compensation is proposed by the work 17 . However, these strategies except this 12 are validated only in the so-called "baseline" pitch control case without any verification in the IPC case. Therefore, this paper proposes a robust unknown input observer (UIO)-based fault estimation (FE) system for pitch sensor fault compensation in the presence of meaurement noise and unmodelled system uncertainties. This strategy is verfied using the LQR-based IPC in the nonlinear, high-fidelity FAST 5MW NREL WT simulator. This is used to complete the proposed fault-tolerant IPC "co-design" scheme.

The remainder of the paper is summarised as follows. The WT structural load analysis and the pitch system mathematical modelling with different sensor faults are presented in Section 2. Following this, Section 3 illustrates the proposed strategies including (i) a robust FE method using UIO and then the pitch sensor fault effects are compensated by a FTC scheme; (ii) an LQR-based IPC approach for both blade and tower asymmetrical loading mitigation. Finally, the detailed simulation results are illustrated in Section 4. Section 5 provides a summary of the work and prospects for further development.

\section{2 | PROBLEM FORMULATION}

The major aim of this paper is to combine FTC techniques with a sustainable controller for offshore WTs in an integrated manner, illustrated in Fig. 1 . Sustainable control here refers to a WT load mitigation scheme using IPC to improve the system sustanability. More sustainability implies enhancing the WT operating life and increasing the percentage of availability. This fault-tolerant individual pitch control "co-design" strategy contains two parts (i) a sustainable pitch controller specifically using the IPC technique to reduce the blade and tower unbalanced loading simultaneously in Region 3, (ii) enhance the load reduction performance in either the fault-free or pitch sensor faulty case and compensate the fault effects using UIO-based FTC method. Therefore, the cost of O\&M will be significantly reduced as well as the reliability and sustanability of offshore WTs are enhanced.

\subsection{Wind Turbine Structual Load Mitigation}

It has been demonstrated that the tower fore-aft bending couples with the blade flap-wise bending moments as well as the tower side-side bending interconnects with the blade edgewise bending moments. The tower fore-aft and side-side bending moments contribute to most of the tower bottom fatigue damage, of which the contribution of tower fore-aft bending exceeds $99 \%$. Therefore, the analysis in this paper focuses on the damping of first flap-wise bending moments and first fore-aft tower vibrations together using multivariable control design.

Generally, different control structures (in terms of choice of actuators, e.g. pitch only or combined pitch and generator) result in various combinations of effective forces acting on the tower top. Table. 1 shows the various possibilities of WT structural load mitigation, depending on actuator strategies 1, 3, 4, 20, 21, 22, In Table. 1 $\times$ means the this control-loop is inactive and the corresponding control variant cannot be used to achieve the load reduction of the corresponding column elment, $\sqrt{ }$ denotes the opposite. Unbalanced blade flap-wise bending moments refer to the tilt and yaw moments 23,24 , which are the main source of blade asymmetrical loading. This imbalance can only be achieved using the disimilar redundancy of the IPC actuation system.

An extra generator torque can be used to mitigate the tower side-side mode from the tower side-side acceleration 22. The most general approach for the standard tower load mitigation controller is to design a decentralized feedback loop with the measured tower top fore-aft oscillations, this is referred to as active tower damping 20 . From Table. 1. we can see that the traditional active tower damping for tower fore-aft bending moments 


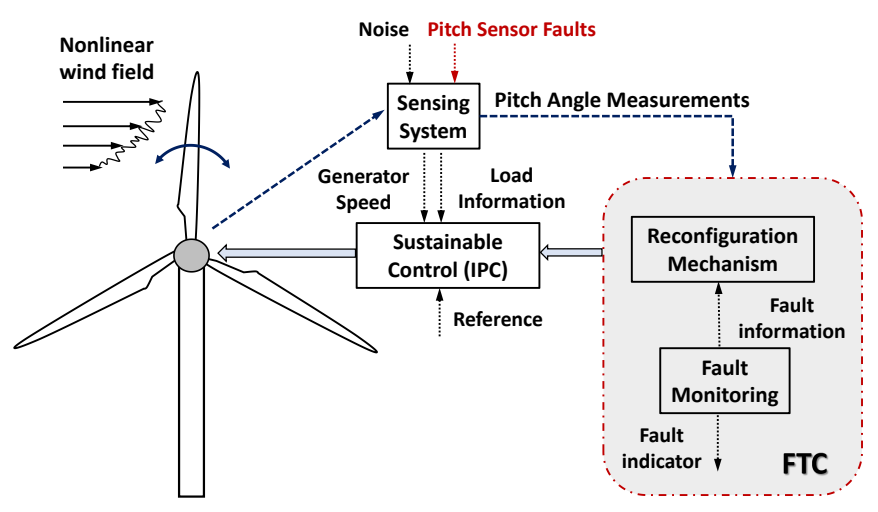

FIGURE 1 Fault-tolerant individual pitch control "co-design" scheme

TABLE 1 Different variants for wind turbine structural load mitigation 1, 3, 4, 20, 21, 22.

\begin{tabular}{cccc}
\hline Controlled Variant & Unbalanced Blade Flap-wise Bending & Tower Fore-aft Bending & Tower Side-side Bending \\
\hline Collective Pitch Angle & $\times$ & $\sqrt{ }$ & $\times$ \\
Individual Pitch Angle & $\sqrt{ }$ & $\sqrt{ }$ & $\sqrt{ }$ \\
Generator Torque & $\times$ & $\times$ & $\sqrt{ }$ \\
\hline
\end{tabular}

is achieved by obtaining an additional collective pitch angle from the measured tower fore-aft acceleration. The proposed controller output is an additional collective pitch reference, complementary and analogous to the standard pitch angle command from CPC. The effectiveness of this approach requires the tower feedback control loop decoupled from the CPC loop and less significant couplings between the turbine blade flap motion and tower fore-aft motion 25 .

Nonetheless, with the increasing tower height of multi-megawatt offshore WTs, the coupling between the blade flap and tower fore-aft motions becomes more significant. The coupling presents a challenge for achieving acceptable load mitigation performance. It turns out to be important to design a multivariable control strategy that can achieve both the blade load reduction and tower fore-aft loading mitigation. Moreover, according to Table.1 IPC provides three individual pitch angles which together can be designed to mitigate the first blade flap-wise bending moment. At the same time the first tower fore-aft and side-side bending moments are mitigated simultaneously using the feedback from the (assumed) fault-free measurements. The measurements are the tower top fore-aft and side-side accelerations and flap-wise bending moments.

This is achieved by the action of dissimilar actuator redundancy which is present in the IPC case as the dissimilar pitch settings effectively balance the rotor. Clearly, the mitigation of unbalanced rotor bending moments cannot be achieved using CPC in which the pitch angles are identical. Therefore, this paper focuses on proposing a multivariable IPC scheme for the fundamental frequency responses of both the tower fore-aft and blade flap-wise bending moments considered together. It is important to note that the frequency band of the extra pitch angle fluctuations for load alleviation should be kept different from the bandwidth of the generator speed control-loops (CPC).

\subsection{Pitch System Modelling with Sensor Faults}

\subsection{1 | Pitch System Sensor Faults}

The WT pitch actuator is adjusted by an internal pitch controller (CPC, or the combination of CPC and IPC) and equipped with pitch sensors for the end pitch angle position feedback of each of the rotor blade, shown in Fig. 2 Using the simulated sensor measurementsrom, the pitch control system guarantees that each rotor blade is in the required reference position, thereby completing the required closed-loop control action. Pitch sensors require a high level of reliability (through redundancy) and should provide accurate blade position feedback, given that the offshore WTs are subject to challenging ambient operating circumstances with constantly changing wind conditions (wind direction, mean wind speed and turbulence intensity). 


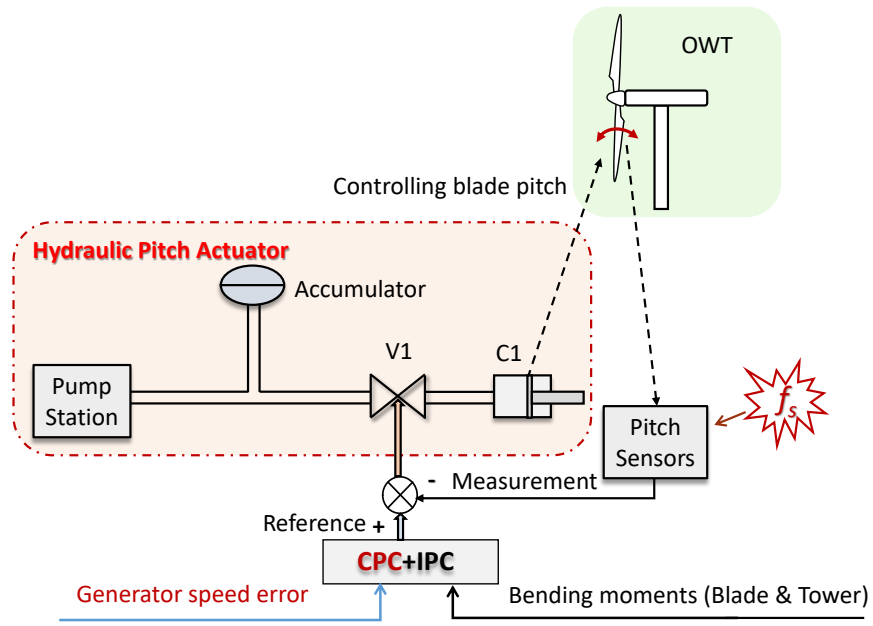

FIGURE 2 Wind turbine hydraulic pitch system

The pitch sensor faults $f_{s}$ are shown to have significant effects on the Region 3 wind turbine performance. Potential pitch sensor faults are caused by a combination of (i) blade pitch bearing maladjustment, (ii) excessive dust on the digital encoder disk, (iii) unreasonable temperature (iv) humidity variations and $(\mathrm{v})$ inaccurate calibration 11 . Either individually or combined these fault components will result in incorrect pitch position readings, further exerting bad effects on the pitch control system. The pitch control system is critical in this role as the optimal pitch reference is based on reliable pitch position measurements. If the sensor faults are not handled correctly, the WT closed-loop dynamics and stability will be affected. Poor closed-loop action and decreased stability will in turn induce an increase in tower and rotor imbalance. Hence, the choice of appropriate FTC scheme considering pitch sensor faults plays a significant role in enhancing the WT sustainability.

The fault effects outlined above can be considered to give rise to the following well known sensor fault classification 13226 :

- Bias sensor fault. This is a common drift fault for analogue sensors belonging to the additive type. The measured sensor reading has an extra constant or time-varying offset caused by sensor temperature variations or calibration issues.

- Stuck with fixed output sensor fault. The sensor output becomes stuck at a constant value and the reading remains fixed after this fault occurs.

- Total sensor fault. This is referred to as a hard sensor fault, which is a form of catastrophic system fault or "failure". A sensor fault manifests itself as a sudden cessation of operation, maintaining a constant zero output, which is often caused by electrical issues (e.g. loss of contact or broken wires) .

- Multiplicative-type (partial) sensor fault. A gain reduction acts on the nominal sensor output and thus the sensor measurement is scaled.

\subsection{2 | Modelling One Pitch System}

The model-based method is adopted here for the pitch sensor fault estimation. Each hydraulic pitch actuator system is modelled as a second-order system with pitch angle range $\left[0^{\circ}, 90^{\circ}\right]$ and pitch rate limits $\left.\left[-8^{\circ} / \mathrm{s}, 8^{\circ} / \mathrm{s}\right]\right]^{18}$, expressed as:

$$
\frac{\beta(s)}{\beta_{r}(s)}=\frac{w_{n}^{2}}{s^{2}+2 \xi w_{n} s+w_{n}^{2}}
$$

where the nominal natural frequency is $\mathrm{w}_{\mathrm{n}}=11.11 \mathrm{rad} / \mathrm{s}$, the damping ratio is $\xi=0.6 . \beta(\mathrm{s})$ is the pitch angle, with $\beta(\mathrm{t})$ the time domain signal. $\beta_{\mathrm{r}}(\mathrm{s})$ is the control reference for the pitch system. The pitch sensor system in the fault-free condition is modelled as:

$$
\beta_{\mathrm{m}}(\mathrm{t})=\beta(\mathrm{t})+\mathrm{d}_{\mathrm{s}}(\mathrm{t})
$$

where $\beta_{\mathrm{m}}(\mathrm{t})$ is the pitch angle measurement, $\mathrm{d}_{\mathrm{s}}(\mathrm{t})$ denotes the measurement noise.

The bias sensor fault is illustrated by adding an additional term $\beta_{\text {bias }}$ to the measured output:

$$
\beta_{\mathrm{m}}(\mathrm{t})=\beta(\mathrm{t})+\beta_{\text {bias }}(\mathrm{t})+\mathrm{d}_{\mathrm{s}}(\mathrm{t})
$$


A fixed sensor output fault means that the measured sensor output maintains a constant value $\beta_{\text {fixed }}$, described as:

$$
\beta_{\mathrm{m}}(\mathrm{t})=\beta_{\text {fixed }}
$$

The total sensor fault is modelled as:

$$
\beta_{\mathrm{m}}(\mathrm{t})=0
$$

The multiplicative sensor fault with gain $f(f=1$ means fault-free) is defined as:

$$
\beta_{\mathrm{m}}(\mathrm{t})=\mathrm{f} \beta(\mathrm{t})+\mathrm{d}_{\mathbf{s}}(\mathrm{t})=\beta(\mathrm{t})+(\mathrm{f}-1) \beta(\mathrm{t})+\mathrm{d}_{\mathrm{s}}(\mathrm{t})
$$

\subsection{3 | Modelling Three Pitch Systems}

It is assumed that each hydraulic pitch system has one pitch sensor and suffers from the same unknown modelling uncertainty $\mathrm{d}(\mathrm{t})$ as the two remaining actuators. Moreover, $\mathrm{d}(\mathrm{t}) \in \mathcal{L}_{2}[0, \infty), \mathrm{d}_{\mathrm{s}}(\mathrm{t}) \in \mathcal{L}_{2}[0, \infty)$, where $\mathcal{L}_{2}$ is the space of square-integrable function. The measurement noise $d_{s}(t)$ is assumed to be the same for each pitch sensor. The pitch sensor fault $f_{s}(t)$ could be negative or positive, and $f_{s}(t)=0$ denotes the fault-free case. Furthermore, the pitch dynamics are assumed to satisfy the following requirements, to guarantee the feasibility of designed UIO.

Assumption 1. The pair $(A, C)$ is observable.

Assumption 2. The faults $\mathrm{f}_{\mathrm{s}}(\mathrm{t})$ are differentiable and thus can be extended into a new (augmented) system state.

Considering the system modelling uncertainty $d$, with measurement noise $d_{s}\left(d_{s} \in \mathbb{R}^{6}\right)$ and sensor faults $f_{s}$, three pitch actuator systems can be modelled as:

where

$$
\begin{aligned}
& \dot{x}(t)=A x(t)+B u(t)+D d(t) \\
& y(t)=C x(t)+F_{s} f_{s}(t)+E_{s} d_{s}(t)
\end{aligned}
$$

$$
f_{s}(t)=\left\{\begin{array}{lr}
\beta_{\text {bias }}, & \text { Biased fault } \\
-\beta(\mathrm{t})+\beta_{\text {fixed }}-\mathrm{d}_{\mathrm{s}}(\mathrm{t}), & \text { Fixed output } \\
-\beta(\mathrm{t})-\mathrm{d}_{\mathrm{s}}(\mathrm{t}), & \text { Total failure } \\
(\mathrm{f}-1) \beta(\mathrm{t}), & \text { Multiplicative fault }
\end{array}\right.
$$

where $\mathrm{x}=\left[\begin{array}{llllll}\dot{\beta_{1}} & \dot{\beta_{2}} & \dot{\beta_{3}} & \beta_{1} & \beta_{2} & \beta_{3}\end{array}\right]^{\top} \in \mathbb{R}^{\mathrm{n}}, \mathrm{u}=\left[\begin{array}{lll}\beta_{\mathrm{r}, 1} & \beta_{\mathrm{r}, 2} & \beta_{\mathrm{r}, 3}\end{array}\right]^{\top} \in \mathbb{R}^{\mathrm{m}}$ and $\mathrm{y}=\left[\begin{array}{lll}\beta_{\mathrm{m}, 1} & \beta_{\mathrm{m}, 2} & \beta_{\mathrm{m}, 3}\end{array}\right]^{\top} \in \mathbb{R}^{\mathrm{p}}$ represent the state variables, control inputs and system outputs, respectively. $\beta_{\mathrm{r}, \mathrm{i}}$ are the $\mathrm{i}^{\text {th }}(\mathrm{i}=1,2,3)$ nominal pitch references, $\beta_{\mathrm{m}, \mathrm{i}}$ are the $\mathrm{i}^{\text {th }}$ nominal pitch angle measurements. $d \in \mathbb{R}^{\prime}$ represents a combined effect of unknown disturbance and modelling uncertainty. $f_{s}=\left[\begin{array}{ll}f_{s 1} & f_{s 2} \\ f_{s 3}\end{array}\right]^{\top} \in \mathbb{R}^{s}$ and $d_{s}=\left[d_{s 1} d_{s 2} d_{s 3}\right]^{\top} \in \mathbb{R}^{r}$ with $d_{s 1}=d_{s 2}=d_{s 3}$ denote assumed sensor faults and measurement noise, respectively. The constant matrices $A \in \mathbb{R}^{n \times n}, B \in \mathbb{R}^{n \times m}, D \in \mathbb{R}^{n \times 1}, C \in$ $\mathbb{R}^{p \times n}, F_{s} \in \mathbb{R}^{p \times s}$, and the sensor noise distribution matrices $E_{s} \in \mathbb{R}^{p \times r}$ are known with $n=6, m=3, I=1, p=3, s=3, r=6$. The parameters of the pitch system model with faults of 7 are illustrated as:

$$
\begin{gathered}
A=\left[\begin{array}{cccccc}
0 & 0 & 0 & 1 & 0 & 0 \\
0 & 0 & 0 & 0 & 1 & 0 \\
0 & 0 & 0 & 0 & 0 & 1 \\
-123.43 & 0 & 0 & -13.332 & 0 & 0 \\
0 & -123.43 & 0 & 0 & -13.332 & 0 \\
0 & 0 & -123.43 & 0 & 0 & -13.332
\end{array}\right], B=\left[\begin{array}{cccc}
0 & 0 & 0 \\
0 & 0 & 0 \\
0 & 0 & 0 \\
123.43 & 0 & 0 \\
0 & 123.43 & 0 \\
0 & 0 & 123.43
\end{array}\right], \\
C=\left[\begin{array}{llllll}
1 & 0 & 0 & 0 & 0 & 0 \\
0 & 1 & 0 & 0 & 0 & 0 \\
0 & 0 & 1 & 0 & 0 & 0
\end{array}\right], D=\left[\begin{array}{l}
0.1 \\
0.1 \\
0.1 \\
0.1 \\
0.1 \\
0.1
\end{array}\right], F_{s}=\left[\begin{array}{lll}
1 & 0 & 0 \\
0 & 1 & 0 \\
0 & 0 & 1
\end{array}\right], E_{s}=\left[\begin{array}{llllll}
1 & 0 & 0 & 0 & 0 & 0 \\
0 & 1 & 0 & 0 & 0 & 0 \\
0 & 0 & 1 & 0 & 0 & 0
\end{array}\right],
\end{gathered}
$$


In this paper, three sensors are assumed to be identical, they have the same probability to suffer from the faults. In order to show clearly the effects of the four different sensor faults, pitch sensor 1 is assumed to suffer from different types of faults $f_{s, 1}$. Pitch sensors 2 and 3 are assumed to be fault-free. The final pitch system with the considered disturbance $d$, sensor fault $f_{s, 1}$ and measurement noise $d_{s}$ is represented in Fig. 3 .

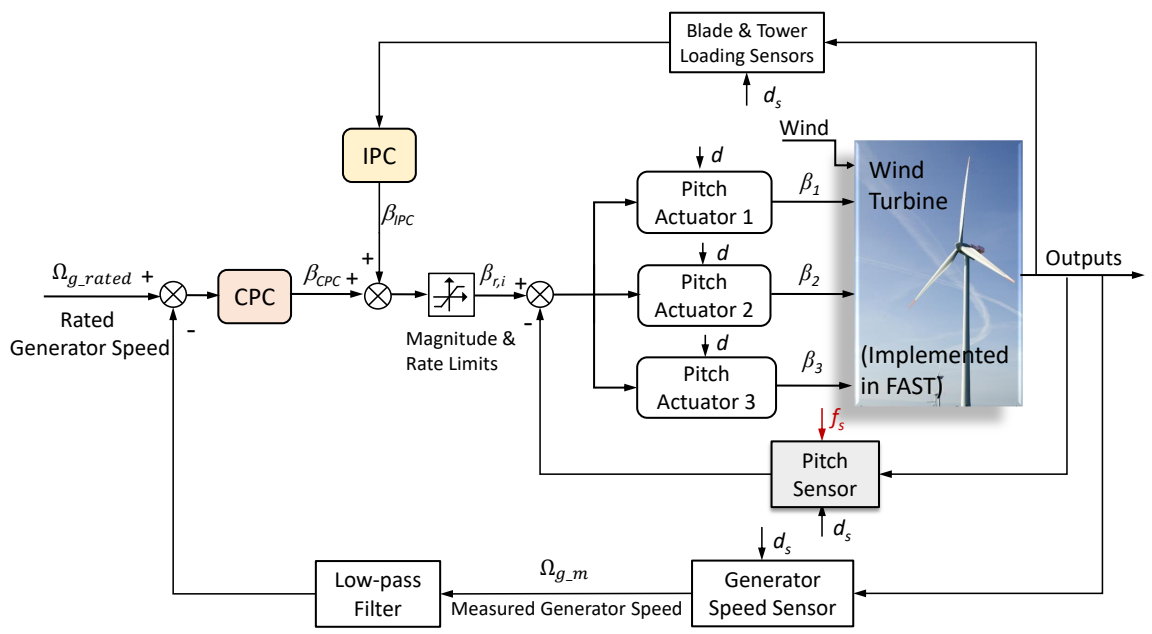

FIGURE 3 Considered pitch system structure with sensor faults

Given that the closed-loop pitch actuator is modelled as a linear system, the error caused by a fault on the blade pitch position measurement moves to the corresponding pitch actuator reference. Therefore, the sensor faults can be modelled as changes in the blade pitch references accordingly, illustrated as:

$$
\beta_{\mathrm{rf}, \mathrm{i}}=\beta_{\mathrm{r}, \mathrm{i}}-\Delta \beta_{\mathrm{m}, \mathrm{i}}, \quad \Delta \beta_{\mathrm{m}, \mathrm{i}}=\beta_{\mathrm{m}, \mathrm{i}}-\beta_{\mathrm{mf}, \mathrm{i}}
$$

where the $\beta_{\mathrm{rf}, \mathrm{i}}$ denotes the new $\mathrm{i}^{\text {th }}$ pitch reference angle containing the sensor fault, $\Delta \beta_{\mathrm{m}, \mathrm{i}}$ means the change of the $\mathrm{i}^{\text {th }}$ pitch angle, $\beta_{\mathrm{mf}, \mathrm{i}}$ is the $i^{\text {th }}$ pitch angle measurement containing the pitch sensor fault.

\section{3 | PROPOSED STRATEGY}

In this Section, the "co-design" framework of the proposed UIO and FTC compensation strategy is shown in Fig. 4 which includes: (i) a baseline pitch controller (CPC) using gain-scheduling proportional-integral for generator power output regulation, and (ii) an LQR-based individual pitch controller for both blade and tower load mitigation, and (iii) a robust UIO-based fault tolerant strategy for pitch sensor fault estimation \& compensation with unknown disturbance and measurement noise. Furthermore, the PI-based IPC controller designed in the previous work ${ }^{27}$ is also considered for better comparison.

The high-fidelity FAST (Fatigue, Aerodynamics, Structure and Turbulence) 5MW NREL WT model with the baseline pitch controller (CPC) and baseline generator torque controller is used as the simulated reference WT ${ }^{19}$. FAST is a nonlinear aero-elastic structural-dynamic model developed by the NREL from the United States for horizontal-axis variable-speed pitch-regulated wind turbines. FAST incorporates the AeroDyn module 28 using the Blade Element Momentum (BEM) theory for the aero-dynamic modelling, which simulates the WT response and loading. FAST is incorporated in the MATLAB/Simulink as an S-Function, so that more flexible access to the turbine control development and design is achievable 29 . The detailed parameters are described in Table.2.

\section{1 | FE-based FTC using Robust UIO}

The pitch system 7 is subject to bounded sensor faults, unknown uncertainties and measurement noise, which will affect the pitch sensor fault estimates. Therefore, a robust FE-based FTC strategy is required to estimate each fault signal effect and achieve the fault compensation to recover 


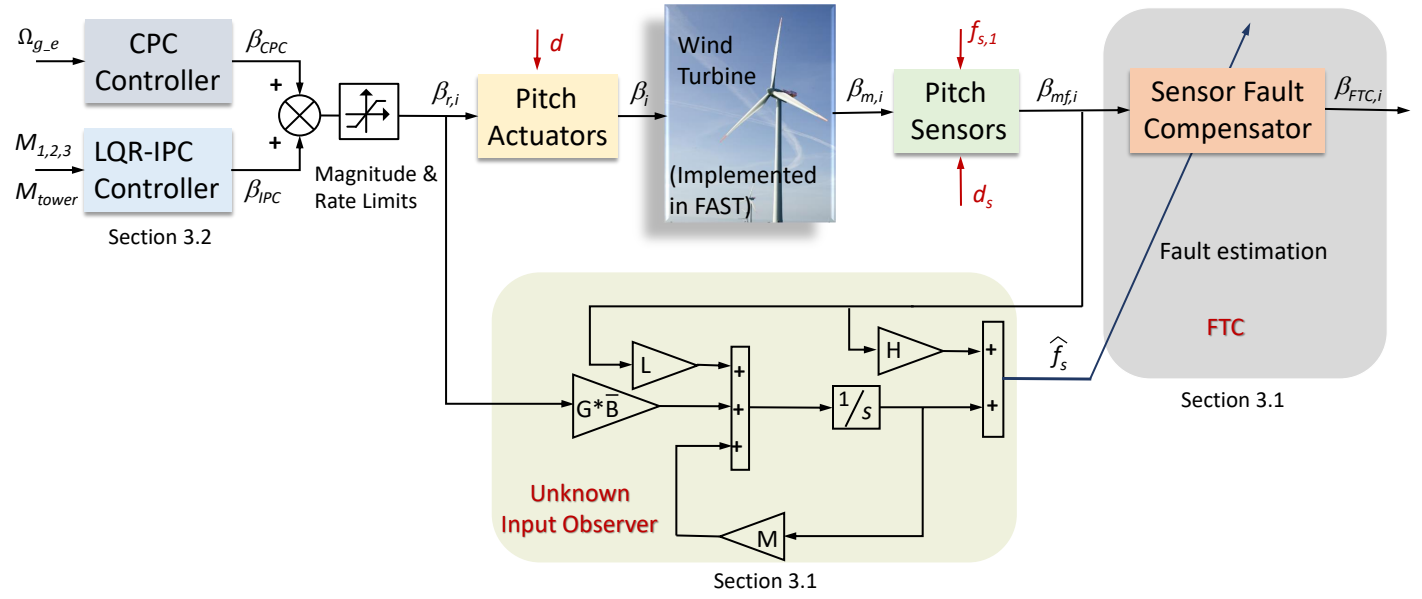

FIGURE 4 Proposed "co-design" framework

TABLE 2 Parameters of the FAST 5MW WT model

\begin{tabular}{ll}
\hline Power rating & $5 \mathrm{MW}$ \\
Rotor orientation, structure & Upwind, three-bladed \\
Rotor, hub diameter & $126 \mathrm{~m}, 3 \mathrm{~m}$ \\
Hub height & $90 \mathrm{~m}$ \\
Cut-in, rated, cut-out wind speed & $3 \mathrm{~m} / \mathrm{s}, 11.4 \mathrm{~m} / \mathrm{s}, 25 \mathrm{~m} / \mathrm{s}$ \\
Rated rotor, generator speed & $12.1 \mathrm{rpm}, 1173.7 \mathrm{rpm}$ \\
Minimum \& maximum pitch angle & $0^{\circ}, 90^{\circ}$ \\
Minimum \& maximum pitch rate & $-8^{\circ} / \mathrm{s}, 8^{\circ} / \mathrm{s}$ \\
\hline
\end{tabular}

the nominal pitch system performance, in the event of sensor faults. The UIO estimator is adopted here because it is not based on knowledge of fault dynamics. The UIO can estimate the fault signals whether or not the fault dynamics are known or unknown. It achieves the fault estimation by including an "unknown input" vector signal $\bar{d}(t)$ comprising the modelling uncertainty $d(t)$ and the first time derivative of the fault signal $\dot{f}_{s}(t)$ as an "augmented" disturbance vector $\overline{\mathrm{d}}(\mathrm{t})=\left[\begin{array}{c}\mathrm{d}(\mathrm{t}) \\ \dot{\mathrm{f}}_{\mathrm{s}}(\mathrm{t})\end{array}\right] . \dot{\mathrm{f}}_{\mathrm{s}}(\mathrm{t})$ is considered as a disturbance or "fault error" signal to be de-coupled by the UIO design. The challenge here is to design a robust UIO-based FTC scheme which uses fault estimation to compensate for the (assumed) sensor fault signal.

For simplicity, the time index is omitted in the following description. To obtain the sensor fault estimates, the first order derivative of the sensor faults $\dot{\mathrm{f}}_{\mathrm{s}}$ is included within an augmented disturbance signal, thus completing the extended pitch system as:

$$
\begin{aligned}
& \dot{\bar{x}}=\bar{A} \bar{x}+\bar{B} u+\bar{D} \bar{d} \\
& y=\bar{C} \bar{x}+E_{s} d_{s}
\end{aligned}
$$

where

$$
\bar{A}=\left[\begin{array}{ll}
\mathrm{A} & 0 \\
\mathbf{0} & \mathbf{0}
\end{array}\right], \bar{B}=\left[\begin{array}{l}
\mathrm{B} \\
\mathbf{0}
\end{array}\right], \bar{D}=\left[\begin{array}{ll}
\mathrm{D} & \mathbf{0} \\
\mathbf{0} & \mathrm{I}
\end{array}\right], \bar{C}=\left[\begin{array}{ll}
\mathrm{C} & \mathrm{F}_{\mathrm{s}}
\end{array}\right], \bar{x}=\left[\begin{array}{l}
\mathrm{x} \\
\mathrm{f}_{\mathrm{s}}
\end{array}\right]
$$

where $\bar{x} \in \mathbb{R}^{n+s}, u \in \mathbb{R}^{m}, y \in \mathbb{R}^{p}, \bar{d} \in \mathbb{R}^{1+s}$ and $\bar{A} \in \mathbb{R}^{(n+s) \times(n+s)}, \bar{B} \in \mathbb{R}^{(n+s) \times m}, \bar{D} \in \mathbb{R}^{n \times(1+s)}, \bar{C} \in \mathbb{R}^{p \times n+s}$.

To estimate the augmented state vector $\bar{x}$, the following UIO structure ${ }^{30}$ is proposed.

$$
\begin{aligned}
& \dot{z}=M z+G \bar{B} u+L y \\
& \hat{x}=z+H y
\end{aligned}
$$


where $z \in \mathbb{R}^{n+s}$ denotes observer states, and $\hat{x} \in \mathbb{R}^{n+s}$ is the estimate of $\bar{x} . M \in \mathbb{R}^{(n+s) \times(n+s)}, G \in \mathbb{R}^{(n+s) \times(n+s)}, L \in \mathbb{R}^{(n+s) \times p}$ and $\mathrm{L} \in \mathbb{R}^{(\mathrm{n}+\mathrm{s}) \times \mathrm{p}}$ are the designed UIO matrices.

Definition 1. The observer 11] is termed as a robust and stable UIO for the pitch system [10, if the state estimation error $e_{x}=\bar{x}-\hat{x}$ asymptotically approaches zero in finite time, in the presence of bounded unknown system disturbance, measurement noise and sensor faults.

Using 10 and 11, the estimation error dynamics are expressed by:

$$
\begin{aligned}
\dot{e}_{x}= & \left(\equiv \bar{A}-L_{1} \bar{C}\right) e_{x} \\
& +\left(\equiv \bar{A}-L_{1} \bar{C}-M\right) z \\
& +\left[\left(\equiv \bar{A}-L_{1} \bar{C}\right) H-L_{2}\right] y \\
& +(\equiv-G) \bar{B} u \\
& +\equiv \bar{D} \bar{d}-L_{1} E_{s} d_{s}-H E_{s} \dot{d}_{s} \\
e_{y}= & \bar{C}_{x}+E_{s} d_{s}
\end{aligned}
$$

where

$$
\begin{aligned}
& \equiv=\mathrm{I}_{\mathrm{n}+\mathrm{s}}-\mathrm{H} \overline{\mathrm{C}} \\
& \mathrm{L}=\mathrm{L}_{1}+\mathrm{L}_{2}
\end{aligned}
$$

To guarantee the proposed observer stability, the following necessary conditions are proposed for the error dynamics 12 :

$$
\begin{aligned}
M \text { is Hurwitz } & \\
\equiv \overline{\mathrm{A}}-\mathrm{L}_{1} \overline{\mathrm{C}}-\mathrm{M} & =0 \\
\left(\equiv \overline{\mathrm{A}}-\mathrm{L}_{1} \overline{\mathrm{C}}\right) \mathrm{H}-\mathrm{L}_{2} & =0 \\
\Xi-\mathrm{G} & =0
\end{aligned}
$$

By satisfying conditions [13-17, the error dynamics [12] become

$$
\begin{aligned}
& \dot{e}_{x}=\left(\equiv A-L_{1} \bar{C}\right) e_{x}+\equiv \bar{D} \bar{d}-L_{1} E_{s} d_{s}-H E_{s} \dot{d}_{s} \\
& e_{y}=\bar{C} e_{x}+E_{s} d_{s}
\end{aligned}
$$

From 18, it can be seen that $\dot{e}_{x}$ is coupled with the disturbance term $\equiv \bar{D} \bar{d}-L_{1} E_{s} d_{s}-H E_{s} \dot{d}_{s}$, which will affect the convergence of $e_{x}$. In other words, if this disturbance term presents a strong coupling between $e_{x}$, the convergence of $e_{x}$ cannot be guaranteed. Thus the FE performance namely the estimation accuracy of the extended state vector $\bar{x}$ will get worse. Therefore, if this disturbance term can be de-coupled from $\dot{e}_{x}$ and all the eigenvalues of $M=\equiv A-L_{1} \bar{C}$ can be assigned to the left half plane of complex plane, $e_{x}$ will converge to zero. From above discussion, it is shown that the measurement noise $d_{s}$ and disturbance $\bar{d}$ in 18 exert negative effects on the state and output estimate errors as well as deteriorate the FE performance. Inspired by the research $\sqrt{31}$, disturbances and measurement noise can be attenuated under a certain level using $\mathrm{H}_{\infty}$ optimization, thus enhancing the UIO robustness and stability.

Theorem 1. If there exists a symmetric positive definite matrix $P \in \mathbb{R}^{(n+s) \times(n+s)}$, and matrices $M_{1} \in \mathbb{R}^{(n+s) \times s}$ and $M_{2} \in \mathbb{R}^{(n+s) \times s}$, such that the error system 18, is robustly stable with $\mathrm{H}_{\infty}$ performance satisfying $\left\|\mathrm{e}_{\mathrm{y}}\right\|_{\infty}<\gamma\left\|\mathrm{w}_{\mathrm{d}}\right\|_{\infty}$ for any disturbance $\mathrm{w}_{\mathrm{d}} \in \mathcal{L}_{2}[0, \infty)$ and a specific constant parameter $\gamma$, then:

$$
\left[\begin{array}{ccccc}
\Pi_{11} & \left(\mathrm{P}-\mathrm{M}_{1} \overline{\mathrm{C}}\right) \overline{\mathrm{D}} & -\mathrm{M}_{2} \mathrm{E}_{\mathrm{s}}+\overline{\mathrm{C}}^{\top} \mathrm{E}_{\mathrm{s}}-\mathrm{M}_{1} \mathrm{E}_{\mathrm{s}} \mathrm{C}^{\top} \\
\star & -\gamma^{2} \mathrm{I} & 0 & 0 & 0 \\
\star & \star & \mathrm{E}_{\mathrm{s}}^{\top} \mathrm{E}_{\mathrm{s}}-\gamma^{2} \mathrm{I} & 0 & 0 \\
\star & \star & \star & -\gamma^{2} \mathrm{I} & 0 \\
\star & \star & \star & \star & -\mathrm{I}
\end{array}\right]<0
$$

where $\Pi_{11}=H_{e}\left(P \bar{A}-M_{1} \bar{C} \bar{A}-M_{2} \bar{C}\right)$, and the disturbance matrix $w_{d}=\left[\bar{d} d_{s} \dot{d}_{s}\right]^{\top} \cdot H_{e}(X)=X+X^{\top}$ and $\star$ represents the transpose of matrix elements in symmetric positions. For the proof of this theorem the reader is referred to the Appendix.

Satisfaction of the LMI 19] ensures the existence of a UIO with stable error dynamics. In addition to this, optimizing the observer dynamic response is also essential to guarantee an acceptable observer performance. It can be achieved by placing all the eigenvalues of the observer 
system matrix within an appropriate sub-domain in the complex plane including discs, vertical strips, conical regions etc. (or combinations thereof) by the LMI optimisation toolbox ${ }^{9}$. To achieve the satisfied closed-loop system time response performance of the proposed UIO, the LMI regional pole placement complement ${ }^{9}$ is adopted to place the observer 19 poles within a suitable vertical strip regions. Here, the observer eigenvalues can be placed to the vertical region $\mathcal{D}: \mathrm{a}<\operatorname{Re}(\lambda)<\mathrm{b}$ with given negative scalars a and $\mathrm{b}(\mathrm{a}<\mathrm{b})$, such that:

$$
\left[\begin{array}{cc}
\mathrm{H}_{\mathrm{e}}\left(\mathrm{P} \overline{\mathrm{A}}-\mathrm{M}_{1} \overline{\mathrm{C}} \overline{\mathrm{A}}-\mathrm{M}_{2} \overline{\mathrm{C}}\right)-2 \mathrm{bP} & 0 \\
\star & -\mathrm{H}_{\mathrm{e}}\left(\mathrm{P} \overline{\mathrm{A}}-\mathrm{M}_{1} \overline{\mathrm{C}} \overline{\mathrm{A}}-\mathrm{M}_{2} \overline{\mathrm{C}}\right)+2 \mathrm{aP}
\end{array}\right]<0
$$

Therefore, by choosing a suitable positive constant $\gamma$, along with negative parameters $a, b$ and solving the LMIs 19 and 20 , $P, M_{1}, M_{2}$ can be achieved. It is worth noting that the matrices $L_{1}$ and $H$ are first obtained with the proposed matrices $P, M_{1}, M_{2}$ according to $H=P^{-1} M_{1}, L_{1}=$ $P^{-1} M_{2}$. Then $M, G, H$ and $L$ can be achieved subsequently with Eq. 13- 17 . Therefore, the sensor fault estimation $\hat{f}_{s}$ can be obtained by the above analysis and design of a robust UIO system.

Finally, the FTC system for fault compensation is realized by subtracting the achieved fault estimate $\hat{\mathrm{f}}_{\mathrm{s}}$ from the faulty pitch measurements $\beta_{\mathrm{mf}}$, shown in:

$$
\beta_{\mathrm{FTC}}=\beta_{\mathrm{mf}}-\hat{\mathrm{f}}_{\mathrm{s}}
$$

where the fault compensation quality is related to the accuracy of the sensor fault estimation $\hat{f}_{s}$, that is whether the estimate error $f_{s}-\hat{f}_{s}$ approaches zero.

\subsection{LQR-based IPC for Blade and Tower Load Mitigation}

The FAST linearisation property $\sqrt{29}$ is carried out under a specific wind condition to achieve the reduced-order linear wind turbine model from the nonlinear 5MW NREL wind turbine simulator for controller design. Here, the linearized wind turbine is obtained under the wind condition with a hub-height mean value $18 \mathrm{~m} / \mathrm{s}$ and a vertical shear exponent of 0.2. Six degrees of freedom (DOFs) including generator rotational flexibility, drive-train rotational mode, first blade flap-wise mode for each blade and first fore-aft tower bending mode are activated. Five outputs including generator speed, flap-wise bending moments (mean, sine and cosine components) and tower fore-aft acceleration are selected as the system outputs. Three individual pitch angles are set as the inputs of this WT system.

The objective function $\mathrm{J}$ in the LQR controller is typically a combination of perturbations of desired states and input variables, parametrized by two weighting matrices (i.e. Q and R). The LQR controller attempts to achieve the optimal control inputs by deriving the algebraic Riccatti equation based on the state-space linear model ${ }^{32}$. In this paper, the LQR controller with assumed full-state feedback is proposed to mitigate the tower first fore-aft bending moment and first blade flap-wise bending moment simultaneously as well as maintain the maximum generator power output.

The control design uses the linear WT model from the FAST linearization process, defined as

$$
\dot{x}(t)=A_{s} x(t)+B_{s} u(t) \quad t \geq 0, x(0)=x_{0}
$$

where $x(t), u(t)$ represent the system state and input vector, respectively. The controllable WT system matrices are $A_{s} \in \mathbb{R}^{11 \times 11}, B_{s} \in \mathbb{R}^{11 \times 3}$. This is a state feedback control problem and the azimuth variable is actually an output of the system and is thus left out of the state variable model formulation. It is required to find the gain matrix $K \in \mathbb{R}^{3 \times 11}$ in the following state feedback control law:

$$
\mathrm{u}(\mathrm{t})=-\mathrm{K} \times(\mathrm{t})
$$

which ensures the closed-loop system is asymptotically stable and minimizes the infinite-time quadratic objective function $\mathrm{J}(\mathrm{t})$ :

$$
J(t)=\int_{0}^{\infty}\left[x^{\top}(t) Q x(t)+u^{\top}(t) R u(t)\right] d t
$$

The selection of $\mathrm{Q}$ and $\mathrm{R}$ matrices plays a significant role in determining the performance of designed load reduction controller. $\mathrm{Q}$ is a positive semi-definite matrix chosen to penalise the system state deviations from the equilibrium (reference values). This can be done by selecting $Q=$ $C_{s}^{\top} Q_{1} C_{s}$, which is appropriate since the system output equation is defined as $y(t)=C_{s} \times(t), C_{s} \in \mathbb{R}^{5 \times 11}$. The matrix $R$ must be invertible and hence positive definite and is chosen to adjust the degree of emphasis on the control input. The larger these parameter values are, the more these signals are penalized. If the parameters in $Q$ are larger, the accurate state (or output) regulation turns out to be more significant and the system allows for more aggressive control, and vice versa. The following LQR design steps ${ }^{32}$ are implemented to achieve the optimal feedback law which satisfies the performance criterion $\mathrm{J}(\mathrm{t})$ :

Step 1: Solve the associated Algebraic Riccati Equation (ARE), return the solution $S$

$$
A_{s}^{\top} S+S A_{s}-S B_{s} R^{-1} B_{s}^{\top} S+Q=0
$$


Step 2: The optimal control matrix $\mathrm{K}$ is derived from $\mathrm{S}$ using

$$
K=R^{-1} B_{s}^{\top} S
$$

Step 3: Hence from [23] and 26, the optimal full-state feedback control law for the WT system is

$$
u^{*}(t)=-R^{-1} B_{s}^{\top} S \times(t)
$$

Different weights $Q_{1}$ and $R$ are tuned to obtain the optimal control performance by trial and error. $Q_{1}=\operatorname{diag}(0.1,1 e-8,6 e-8,6 e-8,0.4)$, $\mathrm{R}=\operatorname{diag}(100,100,100)$ are selected here. In this case, the open-loop and closed-loop eigenvalue locations using LQR of the wind turbine system 22. are shown in Table. 3 and Fig. 5

TABLE 3 Open-loop \& Closed-loop eigenvalue locations

\begin{tabular}{ccc}
\hline DOF & $\begin{array}{c}\text { Open-loop Eigenvalues } \\
(\mathrm{rad} / \mathrm{s})\end{array}$ & $\begin{array}{c}\text { Closed-loop Eigenvalues } \\
(\mathrm{rad} / \mathrm{s})\end{array}$ \\
\hline Generator speed & -0.242 & -0.382 \\
Drive-train first torsion & $-0.734 \pm 14.077 \mathrm{i}$ & $-0.737 \pm 14.073 \mathrm{i}$ \\
First flap-wise blade mean value & $-2.589 \pm 4.046 \mathrm{i}$ & $-3.1057 \pm 4.475 \mathrm{i}$ \\
First flap-wise blade sine component & $-2.654 \pm 4.9924 \mathrm{i}$ & $-3.687 \pm 5.737 \mathrm{i}$ \\
First flap-wise blade cosine component & $-2.644 \pm 2.500 \mathrm{i}$ & $-3.6763 \pm 3.234 \mathrm{i}$ \\
Tower first fore-aft & $-0.149 \pm 2.088 \mathrm{i}$ & $-0.516 \pm 1.877 \mathrm{i}$ \\
\hline
\end{tabular}

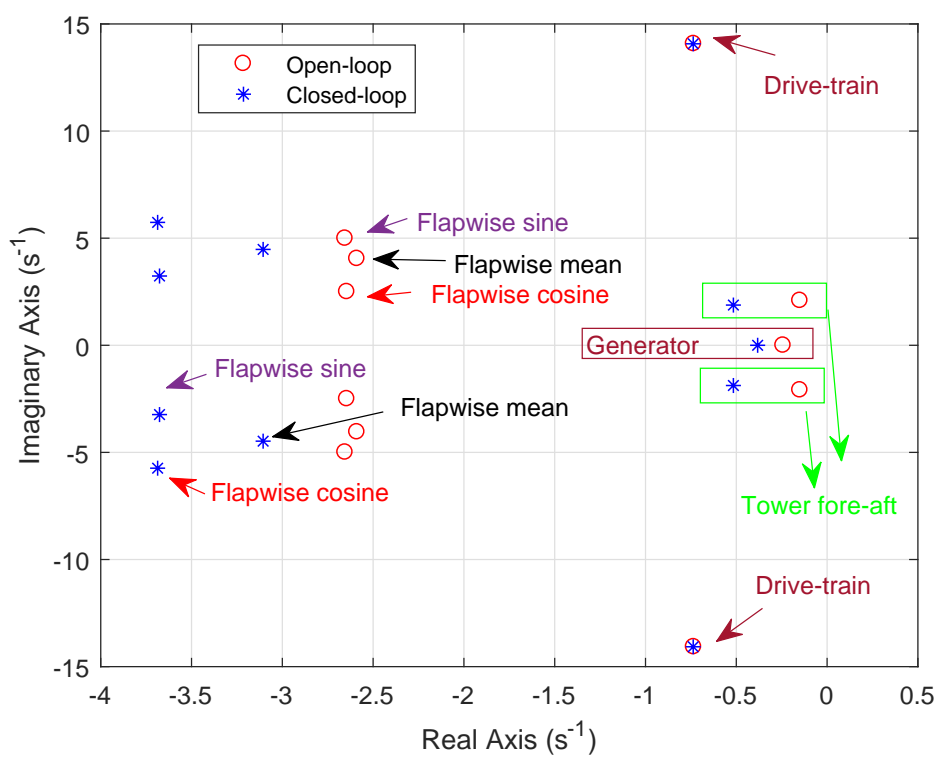

FIGURE 5 Eigenvalue locations of the open-loop and closed-loop wind turbine system (with designed LQR controller)

The feedback design produces increased damping factors for the tower first fore-aft motion and for the blade first bending motion as well as for the generator speed, given that the corresponding real parts of these eigenvalues move further to the left part of the complex plane. Hence, it can be seen that the "damping ratios" of these modes are acceptable, i.e. with only slight oscillation. As this load mitigation problem does not 
include the drive train torsion only a small weighting is placed on the states that relate to the drive-train torsion. The presence of slight oscillation in the bending moments actually presents a challenge to the fault estimation problem since motion with oscillation always has the effect of exciting system nonlinearity and hence uncertainty. The higher the uncertainty the more uncertain will be the fault estimation. Hence, the control design chosen in this work provides a good test for the fault estimation strategy being used.

\section{4 | SIMULATION RESULTS}

In order to consider the mutual effects between pitch sensor faults and the IPC system as well as varied sensor faults, the Turbsim software ${ }^{33}$ is used to give a more faithful representation of wind speed variations and their impact on measurement noise. The simulation correspond to operation above the rated wind speed (Region 3) with the pitch controller active. The three-dimensional turbulent wind fields are obtained based on the IEC Kaimal spectral model along with different turbulence intensities using a normal turbulence model (NTM) and vertical wind shear with power exponent of 0.2. In order to verify the effectiveness of the proposed strategies, two different wind conditions (WCs) in Region 3 are considered as shown in Table 4

TABLE 4 Two different wind conditions, $\mathrm{V}_{\text {mean }}$ is the mean hub-height wind speed, $\mathrm{T}_{\max }$ is the maximum simulation time.

\begin{tabular}{cccc}
\hline WC & $\mathrm{V}_{\text {mean }}(\mathrm{m} / \mathrm{s})$ & Turbulence Intensity (\%) & $\mathrm{T}_{\max }(\mathrm{s})$ \\
\hline WC1 & 18 & 14 & 1000 \\
WC2 & 21 & 16 & 1000 \\
\hline
\end{tabular}

\section{1 | Structural Load Reduction in Fault-free Case}

Simulations are used to compare the LQR-based IPC with both the baseline pitch controller (CPC) and PI-based IPC cases without considering any pitch sensor faults under two wind conditions (i.e. WC1 and WC2). The performance measures includes the standard deviations (STD) of the generator power $\mathrm{P}$, the pitch rate $\dot{\theta}$, the blade 1 flap-wise bending moments $\mathrm{M}_{1}$, the tower fore-aft bending moment $\mathrm{T}_{\mathrm{f}}$ and the tower side-side bending moment $\mathrm{T}_{\mathrm{s}}$. These are calculated from $30 \mathrm{~s}$ to $1000 \mathrm{~s}$ in the simulation to compare these three control strategies. Moreover, the pitch travel $\theta_{\text {sum }}\left(\int_{0}^{\mathrm{t}}|\mathrm{d} \beta / \mathrm{dt}| \mathrm{dt}\right.$ ) is adopted to approximate the pitch actuator movements. Increases in STD and pitch travel denote performance degradation. It is also assumed that the three blades have identical aerodynamic performance. The bending moment STD results have been checked and are seen to be very closely matched for the three blades. Hence, it is only necessary to examine the load mitigation performance of a single blade, labelled blade 1 in the paper. The simulation results in these two different wind conditions in the fault-free case are illustrated in Table 5

As can be seen from Table 5 compared with the CPC case, the proposed LQR-IPC controller can improve the smoothness of the generator power fluctuation by approximately $42.2 \%$ (31.8\% under WC2) whilst the PI-IPC enhances the power vibration by $2.4 \%$ (1.4\% under WC2) with the wind field WC1. Furthermore, the LQR-IPC achieves nearly $24.8 \%$ (25.6\% under WC2) mitigation of the blade flap-wise bending moments as well as $13.3 \%$ reduction ( $24.5 \%$ under WC2) of tower fore-aft bending moments under the wind field WC1. Furthermore, it can be seen that LQR-IPC provides $12.9 \%$ reduction (11.6\% under WC2) of tower side-side bending moments. The PI-based IPC controller achieves a marginally better performance of the mitigation of blade unbalanced loads ( $6 \%$ more compared with LQR-IPC design) but with increased fluctuations of the tower fore-aft (4.1\%) and the side-side bending moments (4.0\%) under WC1. Similar increases occur in the PI-IPC case under WC2. Fig. 6 presents an overview of the simulation results of the three different pitch controllers in the wind condition WC1, which also serves to validate the aforementioned conclusions. Hence, the above results suggest that acceptably robust load reduction performance can be achieved using this LQRbased IPC scheme. It is apparent from Table 5 that both PI-IPC and LQR-IPC controllers represent some similarities with the enhanced pitching motion (not only the pitch rate but also pitch travel) to achieve the goal of structual load reduction, which will inevitably lead to potential pitch system faults.

The frequency analysis of each of the blade 1 flap-wise bending moments $M_{1}$ and tower fore-aft bending moment $T_{f}$ in the wind condition WC1 are shown in Fig.7 It can be seen that the most prominent component of the blade asymmetrical loads is the first harmonic of frequency $1 \mathrm{P}$, which is determined by the rated rotor speed $w_{r}$ (here $w_{r}=12.1 \mathrm{rpm}$, namely $12.1 / 60 \approx 0.2 \mathrm{~Hz}$ ). Meanwhile, the most significant frequency 
TABLE 5 Simulation results in the time domain under two different wind conditions

\begin{tabular}{ccccccc}
\hline Cases & $\mathrm{P}(\mathrm{kW})$ & $\dot{\theta}\left({ }^{\circ} / \mathrm{s}\right)$ & $\theta_{\text {sum }}\left({ }^{\circ}\right)$ & $\mathrm{M}_{1}(\mathrm{kNm})$ & $\mathrm{T}_{\mathrm{f}}(\mathrm{kNm})$ & $\mathrm{T}_{\mathrm{s}}(\mathrm{kNm})$ \\
\hline CPC & $88.8(100 \%)$ & $0.5(100 \%)$ & $380.1(100 \%)$ & $2041.1(100 \%)$ & $7816.8(100 \%)$ & $2867.4(100 \%)$ \\
PI-IPC & $90.9(102.4 \%)$ & $2.5(500.0 \%)$ & $2095.8(551.4 \%)$ & $1410.8(69.1 \%)$ & $8135.7(104.1 \%)$ & $2981.9(104.0 \%)$ \\
LQR-IPC & $51.3(57.8 \%)$ & $2.8(560.0 \%)$ & $2252.1(592.5 \%)$ & $1535.0(75.2 \%)$ & $6778.7(86.7 \%)$ & $2496.8(87.1 \%)$ \\
\hline \multicolumn{7}{c}{$\mathrm{WC2}$} \\
\hline CPC & $139.0(100 \%)$ & $0.8(100 \%)$ & $488.8(100 \%)$ & $2564.7(100 \%)$ & $10801(100 \%)$ & $4988.0(100 \%)$ \\
PI-IPC & $141.0(101.4 \%)$ & $3.1(387.5 \%)$ & $2567.2(525.2 \%)$ & $1810.1(70.6 \%)$ & $10958(101.5 \%)$ & $5586.0(112.0 \%)$ \\
LQR-IPC & $94.8(68.2 \%)$ & $3.5(437.5 \%)$ & $2786.2(570.0 \%)$ & $1907.0(74.4 \%)$ & $8264.4(76.5 \%)$ & $4411.7(88.4 \%)$ \\
\hline
\end{tabular}
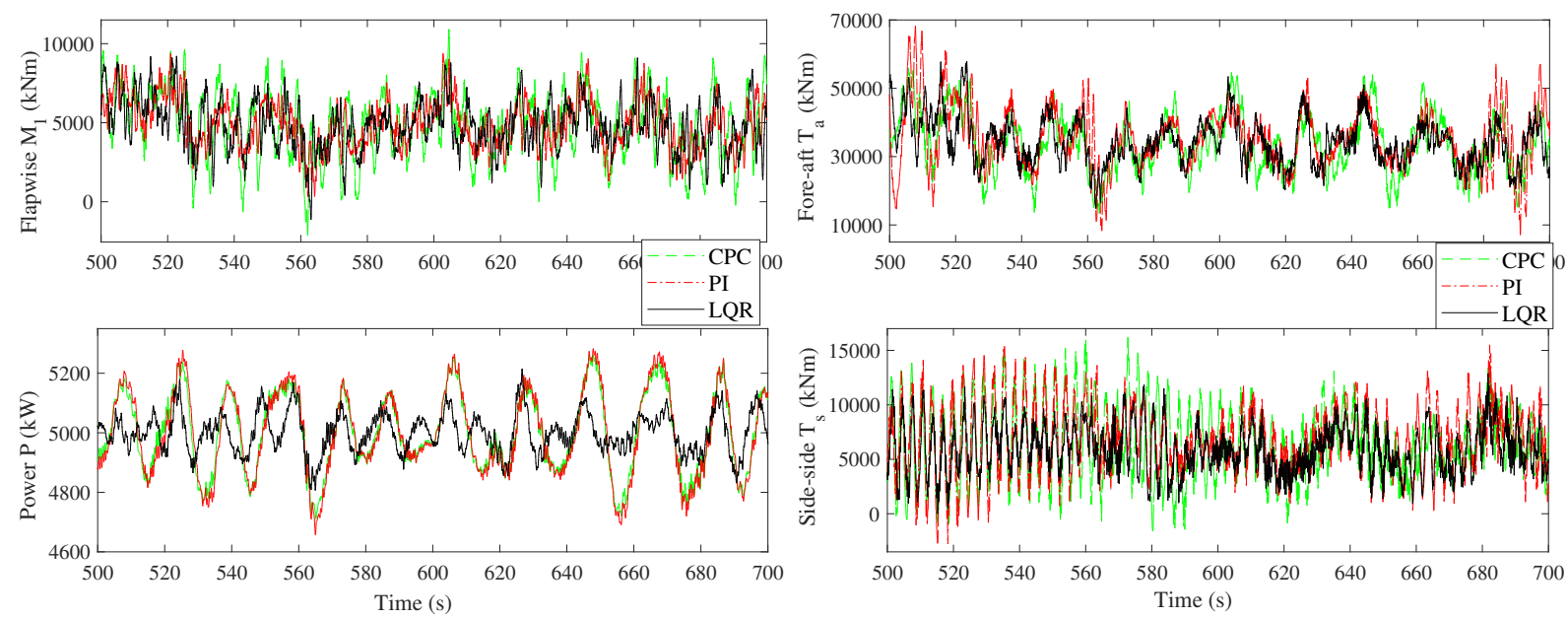

FIGURE 6 Simulation comparison with three different pitch controllers under wind condition WC1

component of the tower fore-aft loading is $0.3 \mathrm{~Hz}$. It is shown that the PI-IPC can obtain better 1P blade loading mitigation than the designed LQRbased IPC, whilst the LQR-IPC can mitigate the unbalanced blade loads around $0.1 \mathrm{~Hz}$. On the other hand, the LQR-IPC controller is observed with notable mitigation of tower fore-aft bending moments near $0.3 \mathrm{~Hz}$.

\section{2 | Fault Estimation Results}

Here, the measurement noise is modelled as white Gaussian Process noise with power $1 * 10^{-6}$. The simulation is carried out under assumed pitch sensor 1 has different types of faults. Pitch senor 2 and 3 are assumed fault-free. The unknown disturbance $d$ and four different fault cases $f_{s 1}$ for blade pitch system sensor 1 are defined as: 

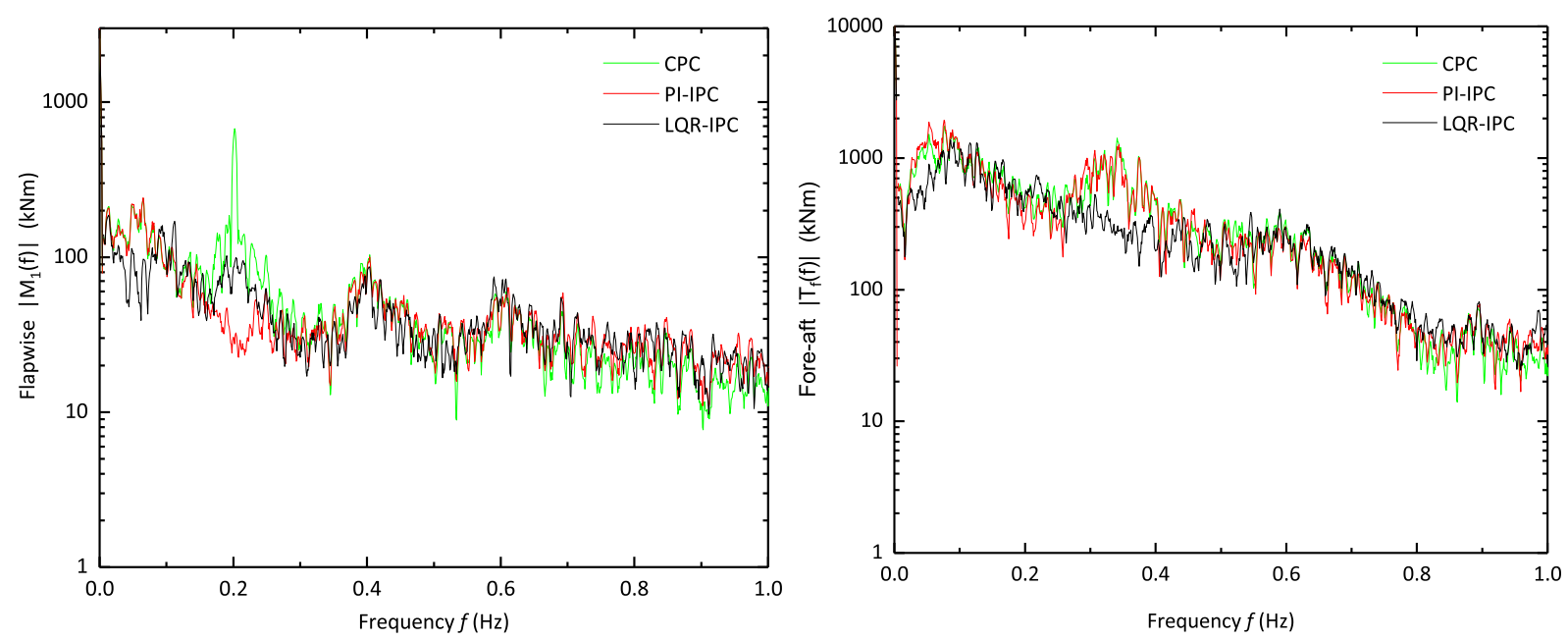

FIGURE 7 Frequency spectrum comparison of $\mathrm{M}_{1}$ and $\mathrm{T}_{\mathrm{f}}$ with three different pitch controllers under wind condition WC1

$$
\begin{aligned}
\mathrm{d}(\mathrm{t}) & =0.01 \sin (\mathrm{t}) \\
\text { Case 1, Changing bias fault: } \mathrm{f}_{\mathrm{s} 1} & =\left\{\begin{array}{lr}
0, & 0 \leq \mathrm{t} \leq 700 \\
5 * \sin (\mathrm{t}), & 700<\mathrm{t} \leq 1000
\end{array}\right. \\
\text { Case 2, Fixed fault at } 14.5^{\circ}: \mathrm{f}_{\mathrm{s} 1} & =\left\{\begin{array}{lr}
0, & 0 \leq \mathrm{t} \leq 700 \\
-\beta(\mathrm{t})+14.5-\mathrm{d}_{\mathrm{s}}(\mathrm{t}), & 700<\mathrm{t} \leq 1000
\end{array}\right. \\
\text { Case 3, Total failure: } \mathrm{f}_{\mathrm{s} 1} & =\left\{\begin{array}{lr}
0, & 0 \leq \mathrm{t} \leq 700 \\
-\beta(\mathrm{t})-\mathrm{d}_{\mathrm{s}}(\mathrm{t}), & 700<\mathrm{t} \leq 1000
\end{array}\right. \\
\text { Case 4, Changing multiplicative gain: } \mathrm{f}_{\mathrm{s} 1} & =\left\{\begin{array}{lr}
0, & 0 \leq \mathrm{t} \leq 700 \\
(-0.0001 \mathrm{t}+0.7-1) \beta(t), & 700<\mathrm{t} \leq 1000
\end{array}\right.
\end{aligned}
$$

The observer gains for the proposed UIO are calculated by solving the proposed LMIs [19] and 20] using the Matlab LMI Toolbox with $\gamma=$ $0.85, a=-80, b=-2.15$. For simplicity and comparison, the sensor fault effects and fault estimation simulation results corresponding to either the CPC or the LQR-IPC pitch controller under wind condition WC1 are illustrated in Figs. 8- 10, The comparison between three pitch measurements in the faulty blade (blade 1) pitch corresponding (1) Sensor bias (2) Fixed output (3) Total failure (4) Multiplicative fault are shown in Fig. 8 and Fig. 9 where the red line is the faulty pitch angle 1 as well as the blue and black lines correspond to the other two pitch angles. The second subfigure of each column in Fig. 8 and Fig. 9 provides more details of the measured pitch angles. It can be seen that the other two healthy pitch angle outputs (i.e. the black and blue lines) are almost same in Fig.8 This is because the CPC pitch controller generates the same pitch angles for the three pitch actuators at the same time instant. In contrast, the other two pitch measurements are different frpm each other due to the existence of the extra pitch angle for load reduction from the LQR-based IPC scheme. From Fig. 8 it is easy to see that the rated pitch angles from the the pitch controllers fluctuate more because of the sensor fault in pitch sensor 1, especially for the fixed sensor output fault case. A similar performance can be seen in the LQR-based IPC case (Fig.9.

The fault estimation results using the designed robust UIO for the considered sensor faults under wind condition WC1 are explained in Fig. 10 and Fig. 11 where the blue line denotes the actual fault signal and the red line denotes the fault estimation result by the proposed UIO method. The second subfigure of each column in Fig. 10 and Fig. 11 provides more details of the pitch sensor fault estimate. Although the UIO was designed using a linear pitch system model, the simulations were performed in a nonlinear aerodynamic wind turbine model. It can be seen from the simulation results that the sensor fault estimation with both pitch controllers show good performance, verifying the robustness of the designed UIO. It is interesting to note that the fault estimates in the CPC case are slightly better than those in the LQR-IPC case. The fault estimates fail to follow some of the high-frequency variations in the LQR-IPC case (see Fig. 11 and to some extent in the CPC case (see Fig. 10. This is the case as extra pitch angles from the designed LQR-IPC have increased the system complexity and impose a strong robustness requirement on the FE scheme. In other words the CPC system has simpler dynamics compared with the IPC case (which is actually a complex combination of both the IPC and CPC). These variations in the LQR-IPC case arise from the effect of the high frequency variations in the combined flap-wise bending and 

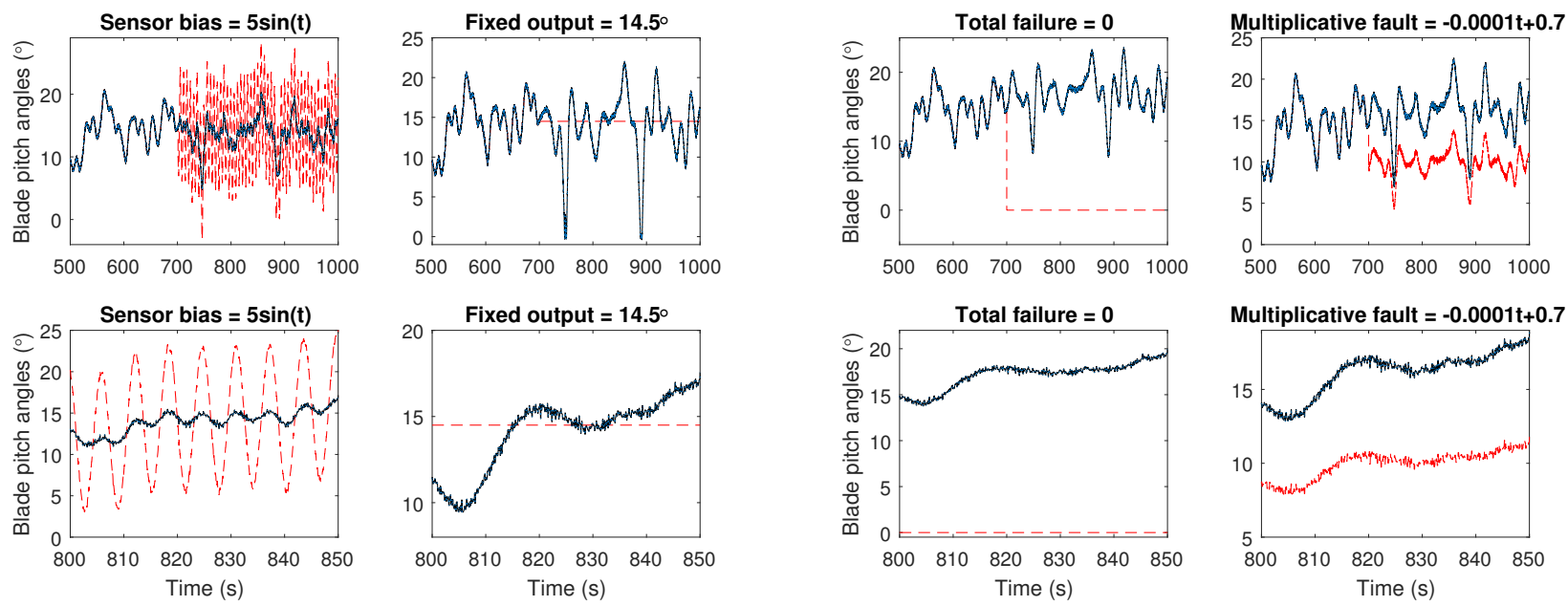

(b)

FIGURE 8 Three pitch measurements from faulty (pitch 1) and fault-free (pitch $1 \& 2$ ) pitch sensors with the CPC pitch controller under wind condition WC1. (a) shows pitch measurements for the cases of either bias or fixed output sensor faults, (b) shows pitch measurements for the case of a total sensor fault (sensor failure) or for a multiplicative sensor fault.
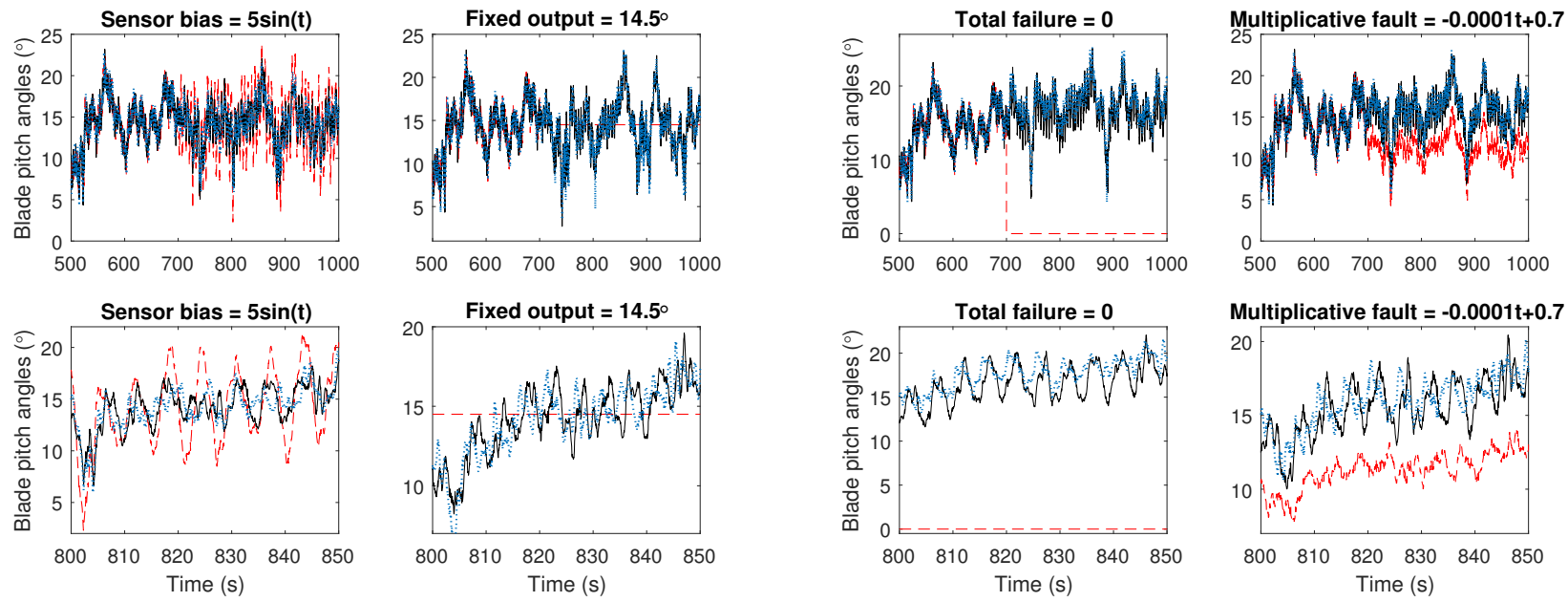

(b)

FIGURE 9 Three pitch measurements from faulty (pitch 1) and fault-free (pitch $1 \&$ 2) pitch sensors with the LQR-based IPC pitch controller under wind condition WC1. (a) shows pitch measurements for the cases of either bias or fixed output sensor faults, (b) shows pitch measurements for the case of a total sensor fault (sensor failure) or for a multiplicative sensor fault.

tower bending moments. This can be seen clearly from the UIO Eq. (11) (see also Fig. 4] which has an input from the flap-wise and tower bending moments via $\beta_{\mathrm{IPC}}$ and the rated pitch angle $\beta_{\mathrm{r}, \mathrm{i}}$. The UIO is designed on the basis of decoupling various "unknown input signals". In this paper, the UIO just considers the disturbance $d$ and measurement noise $d_{s}$ in the pitch system (18). The extra high-frequency pitch angle resulting from the combined blade and tower bending serves as an additional disturbance, which is not considered in the UIO design.

The above figures demonstrate that accurate sensor FE results are achieved in both of the CPC and LQR-IPC cases under the considered two wind conditions. It can be concluded that the UIO designed with $\mathrm{H}_{\infty}$ optimization theory achieves the desired decoupling between the unknown input uncertainties and sensor faults as well as minimising the influence of the measurement noise signals on the FE signal. Hence, good robust performance of the proposed UIO approach is demonstrated in the presence of sensor measurement noise and model disturbance. 

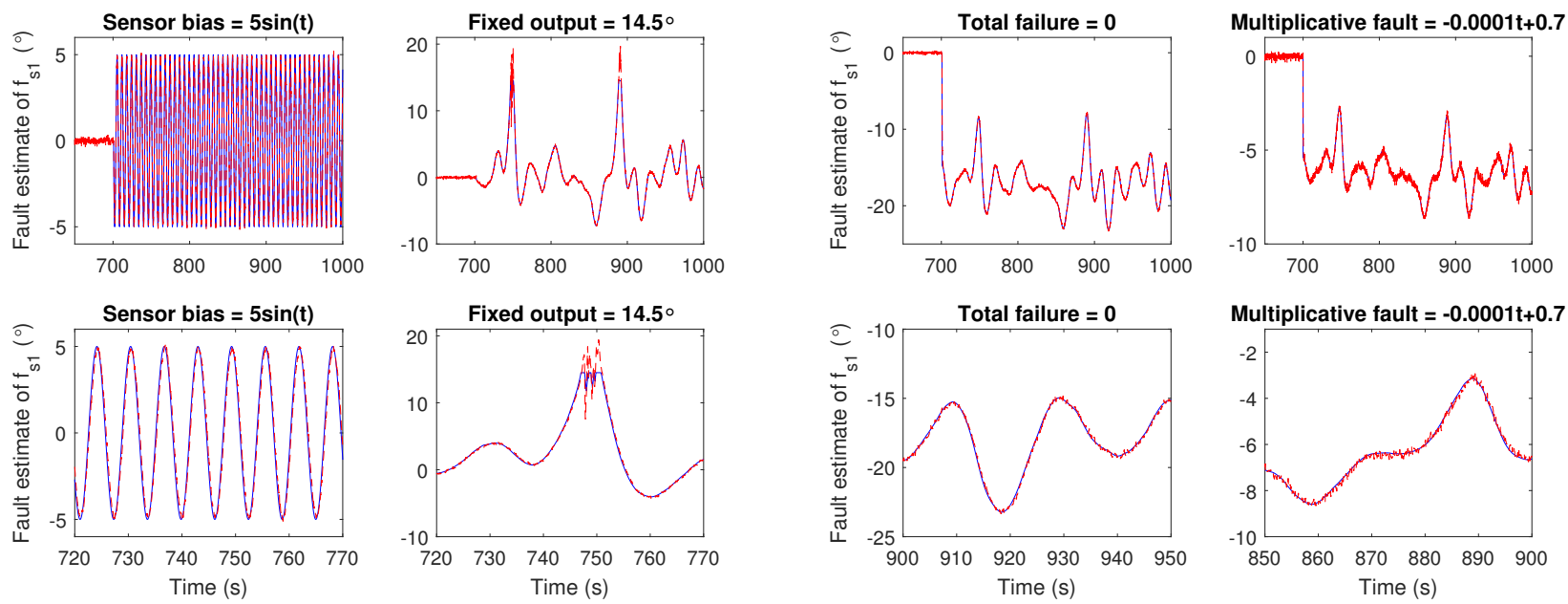

(b)

FIGURE 10 Fault estimation results with the CPC pitch controller under wind condition WC1. (a) shows the estimation of fault $\mathrm{f}_{\mathrm{s} 1}$ for the cases of either bias or fixed output sensor faults, (b) shows the estimation of fault $f_{s 1}$ for the case of a total sensor fault (sensor failure) or for a multiplicative sensor fault.
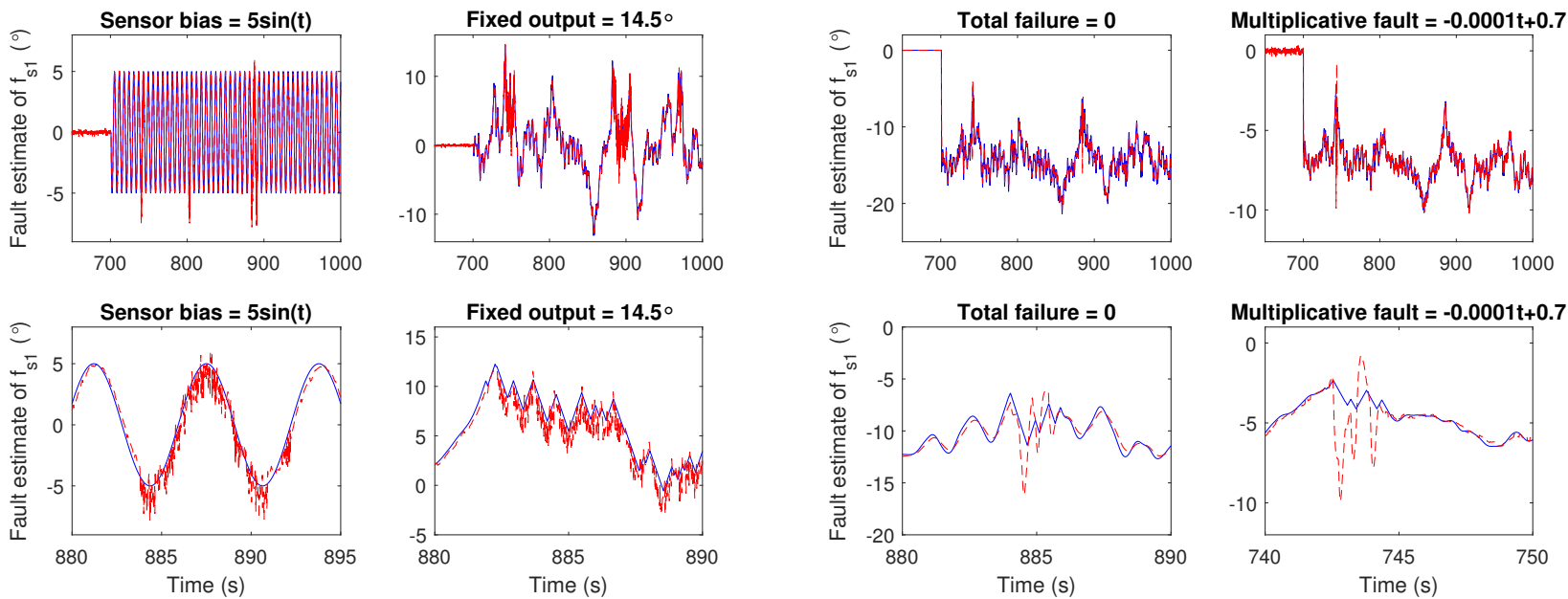

(b)

FIGURE 11 Fault estimation results with the LQR-based IPC pitch controller under wind condition WC1. (a) shows the estimation of fault $\mathrm{f}_{\mathrm{s} 1}$ for the cases of either bias or fixed output sensor faults, (b) shows the estimation of fault $\mathrm{f}_{\mathrm{s} 1}$ for the case of a total sensor fault (sensor failure) or for a multiplicative sensor fault.

\section{3 | Combined Load Reduction and FE-based FTC}

When applying the FE within the UIO strategy, the incorrect measurements are then compensated within the FTC system, validating the design of the proposed FE-based FTC strategy. The simulation results corresponding to 4 different sensor faults with 3 kinds of pitch controllers and related FTC performances in 2 different wind conditions (i.e. 54 cases in total) are illustrated in Table B1. The CPC_bias denotes the baseline pitch controller, considering the bias faults of sensor 1. CPC_bias_FTC means the case of CPC_bias with the designed UIO-based FTC system. Similar explanations follow for the other cases. Furthermore, the corresponding bar graph for the simulation results are illustrated in Fig. 12 providing more insight into the performance of different pitch controllers in the fault-free, faulty without FTC and faulty with FTC cases.

The detailed simulation results under two wind conditions validate the effectiveness of the proposed "co-design" strategy. The results also illustrate clearly the consistency of the corresponding simulation results for the two wind conditions. From Fig. $12 \mathrm{a}$ the LQR-based IPC case is 


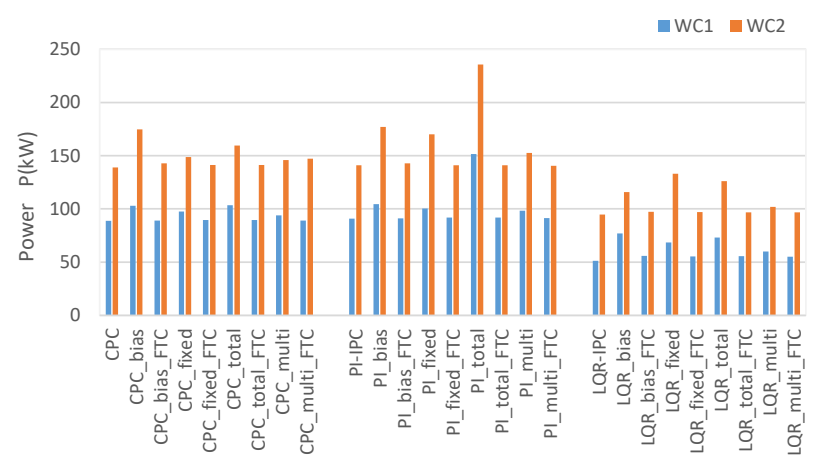

(a)

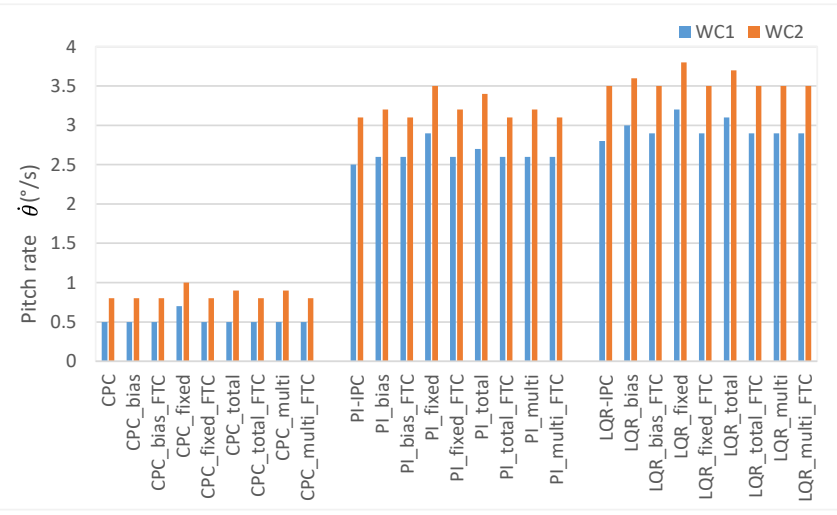

(c)

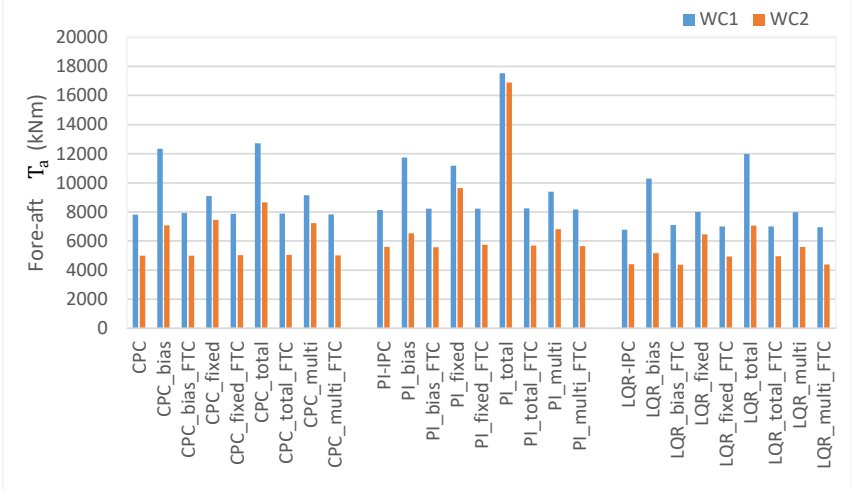

(e)

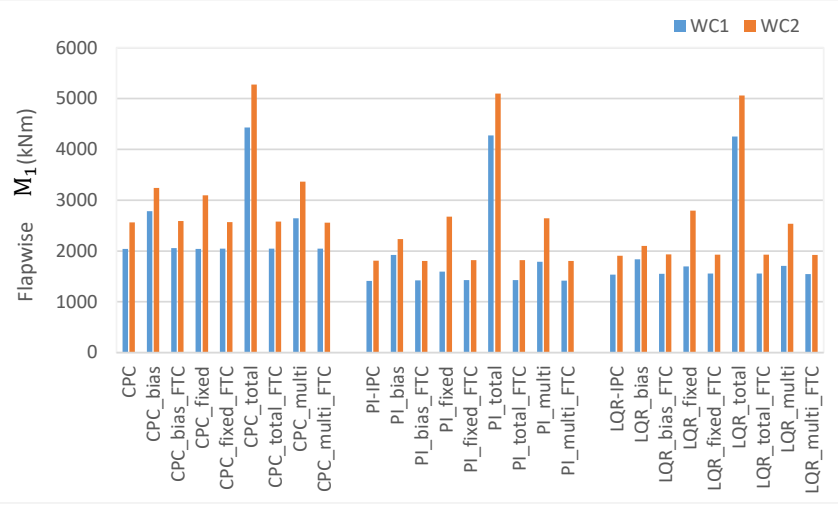

(b)

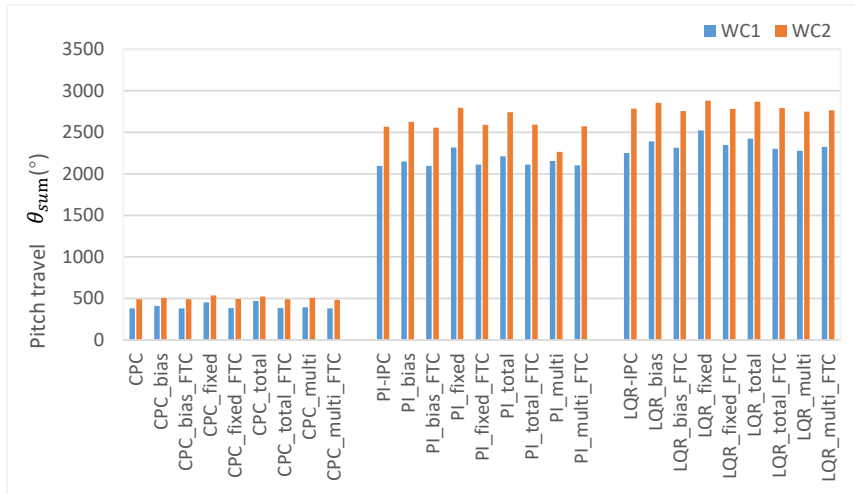

(d)

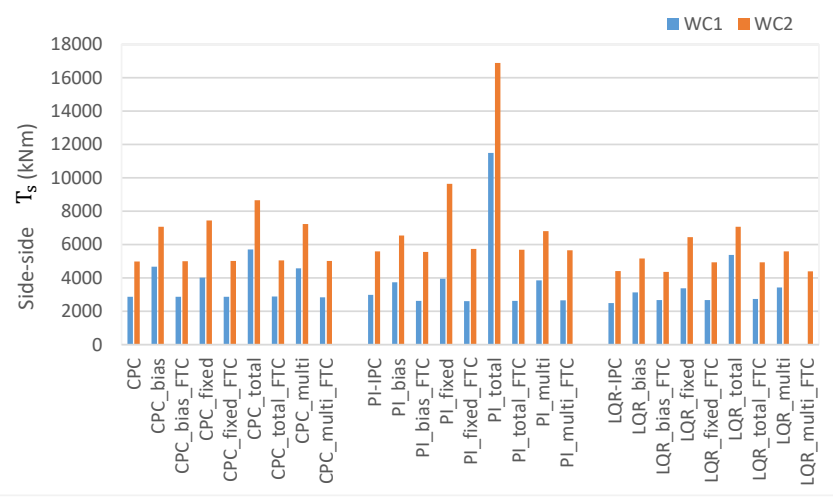

(f)

FIGURE 12 Bar graph of simulation result comparison under 4 different faults of pitch sensor 1 in different pitch controllers under 2 wind conditions

shown to have better performance in terms of reducing the STD of the generator power output, under both wind fields. From Fig. $12 \mathrm{~b}$ the LQRbased IPC have similar performance with the PI-based IPC in terms of blade bending moments in the faulty cases, which are affected significantly by the pitch total sensor fault. From Fig. $12 \mathrm{c}$ and Fig. $12 \mathrm{~d}$ the STD of the pitch rate $\operatorname{STD}(\dot{\theta})$ and the pitch travel $\theta_{\text {sum }}$ have similar trends. When pitch sensor 1 suffers from faults (especially a fixed fault), $\operatorname{STD}(\dot{\theta})$ and $\theta_{\text {sum }}$ increase by different levels. This shows that the pitch actuator 1 has to work harder to achieve the reference pitch angle from the associated pitch controller in the presence of sensor faults. Note that the FE-based FTC scheme can reduce the enhanced pitch rate and motion of pitch actuators due to sensor faults (especially the fixed output and total sensor failure). In this sense, the proposed scheme does not only compensate the fault effects but also has the potential of decreasing the pitch actuator fatigue during the long-term operation, in the event of pitch sensor faults. From Figs. 12e and $12 \mathrm{f}$ it can be seen that the LQR-based IPC can maintain better unbalanced load reduction performance of each of the tower fore-aft and side-side bending moments under both wind conditions, especially in the case of the pitch total sensor fault, which also applies to the other three sensor fault types. Furthermore, it can be seen that 
different sensor faults have varying degrees of impact on the WT system. Considering the fault levels described in this paper, the example of total failure of the pitch sensor shows the greatest influence on the considered wind turbine performance, followed by the fixed sensor output fault. It is necessary to emphasise that no matter what the pitch controller is, the proposed "co-design" strategy can recover the fault-free performance when four types of sensor faults occur, and for the two wind conditions considered. This shows that the load mitigation performance in the fault case is recovered to a similar level when no faults are active in the pitch system. It is clear from the above that the proposed "co-design" strategy not only reduces the turbine unbalanced loading but also reduces the power output fluctuation and the likelihood of pitch actuator fatigue, in the event of pitch sensor faults.

In this way the robustness and effectiveness of the proposed UIO-based FTC strategy are validated. Based on the sensor FE results, the proposed FE-based FTC stategy is feasible and the system has sufficient robustness to compensate the fault effects when different pitch controllers are used. For example, the case of LQR_bias_FTC show similar results to the fault-free LQR-IPC case. Similar results follow for the other cases when using the same FTC design strategy. It can be seen that the simulation results for the cases of LQR_bias_FTC, LQR_fixed_FTC and LQR_multi_FTC after the fault compensation are marginally worse than the baseline LQR-IPC case. This is due to the fact that the FE results for these three cases are not as accurate as in other cases. Recall that the quality of the designed FTC scheme relies on the accuracy of the FE signals generated by the UIO system. However, improved load reduction performance comes with the cost of higher pitch rates. The increased pitch usage is still reasonable in the LQR-IPC case, given that the additional pitch angles are required to mitigate both the tower loading and the rotor asymmetrical loading.

The work shows that alternative IPC strategies present different load reduction performance in the presence of pitch sensor faults. On the whole, the LQR-based IPC system demonstrates an improved performance compared with the PI-based IPC. This is measured in terms of fault-tolerant load mitigation performance, when considering the combined problem of reduction of blade and tower bending moments. The LQR-based IPC system also attenuates the negative effects of pitch sensor faults on the turbine structural loading and on the power output. What has been learnt is that it is important to consider the predicted performance of the load reduction controller at the design stage of the fault-tolerant IPC system, for a range of typical sensor fault cases. Hence, at an early stage of wind turbine rotor and tower system development it is important to consider the component reliability but also a stratgy for fault-tolerance that is consistent with expected control actions and performance. One of the driving issues of this is to determine how the control actions and fault tolerance can be designed together, taking into account the important function of unbalanced load mitigation. The combination of these design issues at an early stage can be referred to as a fault-tolerant IPC "co-design" scheme.

\section{$5 \quad$ CONCLUSION}

This paper proposes a "co-design" idea of considering all the "fault effects" together acting in the both rotor and tower system containing the structual bending moments and pitch sensor faults. The following contributions have been made:

(i) The proposal of an LQR-based IPC control strategy for mitigating the blade asymmetrical loads and tower fore-aft loading simultaneously, and

(ii) A robust UIO-based FTC system combined with $\mathrm{H}_{\infty}$ optimization theory for accurate sensor fault estimation and compensation is designed with one pitch sensors employed in each actuator. This design also ensures decoupling of the effects of unknown system disturbance and measurement noise from the FE signal. Finally,

(iii) The designed IPC system and sensor FE-based FTC strategy are combined together to validate the robustness and achieve a comprehensive performance evaluation of the proposed strategy.

It is therefore concluded that the proposed LQR-based IPC system has a better performance than the PI-based IPC not only in mitigating the tower and blade unbalanced loading and power output fluctuations in both fault-free operation and when pitch sensor faults occur. The chosen simulation strategy provides a useful way of demonstrating the applicability of the pitch system fault-tolerant IPC using LQR-based IPC with fault estimation subsystem. It is worth noting that the "co-design" strategy considers the reduction of unblanced blade and tower loads as well as mitigating the pitch actuator fatigue by recovering nominal pitch rates and actuator movements in the presence of pitch sensor faults. It is important to note that when a fault or faults occur in the pitch system the load mitigation performance cannot be recovered without the use of the on-line management of the sensor faults. Finally, this work has the potential of offering a way to enhance the effectiveness of O\&M procedures and hence enhancing the WT sustainability and maintaining good levels of LCoE. Future work will include an investigation of robustness arising from the combined/integrated robustness of the estimation and control actions when considering joint unbalanced load mitigation and pitch faults.

\section{ACKNOWLEDGEMENTS}

The authors thanks the China Scholarship Council and the Hull-China Scholarship for PhD funding support for Yanhua Liu. Thanks are also expressed to the UK EPSRC for funding support in the project "A New Partnership in Offshore Wind" through grant ref EP/R004900/1. 


\section{APPENDIX}

\section{A PROOF OF THEOREM 1}

Proof. Consider the following Lyapunov function $\mathrm{V}=\mathrm{e}_{x}^{\top} \mathrm{P} \mathrm{e}_{\mathrm{x}}$ and $\mathrm{P}$ is a symmetric positive definite matrix. Combined with the error dynamics 18, the first time derivative of $\mathrm{V}$ is represented as:

$$
\begin{aligned}
& \dot{\mathrm{V}}=\dot{\mathrm{e}}_{\mathrm{x}}^{\top} \mathrm{P} \mathrm{e}_{\mathrm{x}}+\mathrm{e}_{\mathrm{x}}^{\top} \mathrm{P} \dot{\mathrm{e}}_{\mathrm{x}} \\
& =e_{x}^{\top} M^{\top} P e_{x}+\bar{d}^{\top} \bar{D}^{\top} \equiv^{\top} P e_{x}-d_{s}^{\top} E_{s}^{\top} L_{1}^{\top} P e_{x} \\
& -\dot{d}_{s}^{\top} E_{s}^{\top} H^{\top} P e_{x}+e_{x}^{\top} P M e_{x}+e_{x}^{\top} P \equiv \bar{D} \bar{d} \\
& -e_{x}^{T} P L_{1} E_{s} d_{s}-e_{x}^{T} P H E_{s} d_{s} \\
& =\left[\begin{array}{c}
e_{x} \\
\bar{d} \\
d_{s} \\
\dot{d}_{s}
\end{array}\right]^{\top}\left[\begin{array}{cccc}
M^{\top} P+P M & P \equiv \bar{D} & -P_{1} E_{s} & -P H E_{s} \\
\star & 0 & 0 & 0 \\
\star & \star & 0 & 0 \\
\star & \star & \star & 0
\end{array}\right]\left[\begin{array}{c}
e_{x} \\
\bar{d} \\
d_{s} \\
\dot{d}_{s}
\end{array}\right]
\end{aligned}
$$

The $\mathrm{H}_{\infty}$ performance of the output estimation error $\left\|\mathrm{e}_{\mathrm{y}}\right\|_{\infty}<\gamma\left\|\mathrm{w}_{\mathrm{d}}\right\|_{\infty}$, which aims to make the estimation error insensitive to the disturbance matrix and illustrated as

$$
J=\int_{0}^{\infty}\left(e_{y}^{\top} e_{y}-\gamma^{2} w_{d}^{\top} w_{d}\right) d t<0
$$

To achieve the performance required by [A2], one sufficient condition is shown as:

$$
\mathrm{J}_{1}=\mathrm{e}_{\mathrm{y}}^{\top} \mathrm{e}_{\mathrm{y}}-\gamma^{2} \mathrm{w}_{\mathrm{d}}^{\top} \mathrm{w}_{\mathrm{d}}+\dot{\mathrm{V}}<0
$$

The sufficiency proof of $\mathrm{A} 3$ is shown as follows. With assumed zero initial conditions, then it holds that:

$$
\begin{aligned}
J & =\int_{0}^{\infty}\left(e_{y}^{\top} e_{y}-\gamma^{2} w_{d}^{\top} w_{d}\right) d t \\
& =\int_{0}^{\infty}\left(e_{y}^{\top} e_{y}-\gamma^{2} w_{d}^{\top} w_{d}+\dot{V}\right) d t-\int_{0}^{\infty} \dot{V} d t \\
& =\int_{0}^{\infty}\left(e_{y}^{\top} e_{y}-\gamma^{2} w_{d}^{\top} w_{d}+\dot{V}\right) d t-V(\infty)+V(0) \\
& =\int_{0}^{\infty}\left(e_{y}^{\top} e_{y}-\gamma^{2} w_{d}^{\top} w_{d}+\dot{V}\right) d t-V(\infty)
\end{aligned}
$$

Given that the Lyapunov function $\mathrm{V}$ is nonnegative, therefore the following inequality holds:

$$
J \leq \int_{0}^{\infty}\left(e_{y}^{\top} e_{y}-\gamma^{2} w_{d}^{\top} w_{d}+\dot{V}\right) d t
$$

If $\mathrm{J}_{1}=\mathrm{e}_{\mathrm{y}}^{\top} \mathrm{e}_{\mathrm{y}}-\gamma^{2} \mathrm{w}_{\mathrm{d}}^{\top} \mathrm{w}_{\mathrm{d}}+\dot{\mathrm{V}}<0$ is satisfied, therefore its integration $\int_{0}^{\infty}\left(e_{y}^{\top} e_{y}-\gamma^{2} w_{d}^{\top} w_{d}+\dot{\mathrm{V}}\right) d t<0$, so that $\mathrm{J}<0$ is guaranteed.

Combining A1] with A3, the following formula can be obtained:

$$
\left[\begin{array}{c}
e_{x} \\
\bar{d} \\
d_{s} \\
\dot{d}_{s}
\end{array}\right]^{T}\left[\begin{array}{cccc}
M^{\top} P+P M+\bar{C}^{\top} \bar{C} P \equiv \bar{D} & -P_{1} E_{s}+\bar{C}^{\top} E_{s}-P H E_{s} \\
\star & -\gamma^{2} \mathrm{I} & 0 & 0 \\
\star & \star & E_{s}^{\top} E_{s}-\gamma^{2} I & 0 \\
\star & \star & \star & -\gamma^{2} I
\end{array}\right]\left[\begin{array}{c}
e_{x} \\
\bar{d} \\
d_{s} \\
\dot{d}_{s}
\end{array}\right]<0
$$


Note that the LMI A5 is not linear with respect to the variables $\mathrm{P}, \mathrm{L}_{1}$ and $\mathrm{H}$, therefore the reformulation $\mathrm{M}_{1}=\mathrm{PH}_{,} \mathrm{M}_{2}=\mathrm{PL}_{1}$ are defined for convenience. With the use of Schur Complement Lemma ${ }^{34}$, the following LMI equation can be achieved:

$$
\left[\begin{array}{ccccc}
\mathrm{He}\left(\mathrm{P} \overline{\mathrm{A}}-\mathrm{M}_{1} \overline{\mathrm{C}} \overline{\mathrm{A}}-\mathrm{M}_{2} \overline{\mathrm{C}}\right) & \left(\mathrm{P}-\mathrm{M}_{1} \overline{\mathrm{C}}\right) \overline{\mathrm{D}} & -\mathrm{M}_{2} \mathrm{E}_{\mathrm{s}}+\overline{\mathrm{C}}^{\top} \mathrm{E}_{\mathrm{s}} & -\mathrm{M}_{1} \mathrm{E}_{\mathrm{s}} & \mathrm{C}^{\top} \\
\star & -\gamma^{2} \mathrm{I} & 0 & 0 & 0 \\
\star & \star & \mathrm{E}_{\mathrm{s}}^{\top} \mathrm{E}_{\mathrm{s}}-\gamma^{2} \mathrm{I} & 0 & 0 \\
\star & \star & \star & -\gamma^{2} \mathrm{I} & 0 \\
\star & \star & \star & \star & -\mathrm{I}
\end{array}\right]<0, P>0
$$

This completes the proof of Theorem 1.

\section{B SUMMARY OF SIMULATION RESULTS UNDER TWO WIND CONDITIONS}

TABLE B1 Summary of simulation results under 4 different pitch sensor 1 faults in different pitch controllers under 2 wind conditions

\begin{tabular}{|c|c|c|c|c|c|c|c|c|c|c|c|c|}
\hline \multirow[b]{2}{*}{ Cases } & \multicolumn{6}{|c|}{ WC1 } & \multicolumn{6}{|c|}{ WC2 } \\
\hline & $\mathrm{P}(\mathrm{kW})$ & $\dot{\theta}(\mathrm{\circ} / \mathrm{s})$ & $\theta_{\text {sum }}\left({ }^{\circ}\right)$ & $\mathrm{M}_{1}(\mathrm{kNm})$ & $\mathrm{T}_{\mathrm{f}}(\mathrm{kNm})$ & $\mathrm{T}_{\mathrm{s}}(\mathrm{kNm})$ & $P(k W)$ & $\dot{\theta}(\mathrm{\circ} / \mathrm{s})$ & $\theta_{\text {sum }}\left({ }^{\circ}\right)$ & $\mathrm{M}_{1}(\mathrm{kNm})$ & $\mathrm{T}_{\mathrm{f}}(\mathrm{kNm})$ & $\mathrm{T}_{\mathrm{s}}(\mathrm{kNm})$ \\
\hline CPC & 88.8 & 0.5 & 380.1 & 2041.1 & 7816.8 & 2867.4 & 139.0 & 0.8 & 488.8 & 2564.7 & 10801.0 & 4988.0 \\
\hline PI-IPC & 90.9 & 2.5 & 2095.8 & 1410.8 & 8135.7 & 2981.9 & 141.0 & 3.1 & 2567.2 & 1810.1 & 10958.0 & 5586.0 \\
\hline LQR-IPC & 51.3 & 2.8 & 2252.1 & 1535.0 & 6778.7 & 2496.8 & 94.8 & 3.5 & 2786.2 & 1907.0 & 8264.4 & 4411.7 \\
\hline CPC_bias & 102.9 & 0.5 & 411.5 & 2786.1 & 12349.0 & 4678.1 & 174.8 & 0.8 & 507.0 & 3238.8 & 14266.0 & 7069.2 \\
\hline CPC_bias_FTC & 89.1 & 0.5 & 381.0 & 2056.5 & 7923.8 & 2873.3 & 142.9 & 0.8 & 489.2 & 2588.7 & 10816.0 & 4998.1 \\
\hline CPC_fixed & 97.6 & 0.7 & 454.1 & 2038.7 & 9097.0 & 4021.2 & 148.6 & 1.0 & 535.1 & 3096.6 & 11445.0 & 7453.2 \\
\hline CPC_fixed_FTC & 89.7 & 0.5 & 382.7 & 2047.8 & 7870.5 & 2877.6 & 141.2 & 0.8 & 493.1 & 2569.1 & 10869.0 & 5024.7 \\
\hline CPC_total & 103.5 & 0.5 & 471.4 & 4428.8 & 12711 & 5711.1 & 159.5 & 0.9 & 523.0 & 5274.8 & 16112.0 & 8654.3 \\
\hline CPC_total_FTC & 89.7 & 0.5 & 382.8 & 2048.0 & 7884.8 & 2887.1 & 141.3 & 0.8 & 489.3 & 2581.8 & 10885.0 & 5052.2 \\
\hline CPC_multi & 93.9 & 0.5 & 393.2 & 2643.5 & 9145.8 & 4579.1 & 145.9 & 0.9 & 505.3 & 3367.5 & 12416.0 & 7230.1 \\
\hline CPC_multi_FTC & 89.1 & 0.5 & 381.0 & 2045.4 & 7832.9 & 2840.1 & 147.3 & 0.8 & 484.6 & 2560.3 & 10671.0 & 5015.1 \\
\hline PI_bias & 104.6 & 2.6 & 2149.2 & 1924.2 & 11743.0 & 3742.1 & 177.0 & 3.2 & 2628.3 & 2235.7 & 13871.0 & 6539.8 \\
\hline PI_bias_FTC & 91.2 & 2.6 & 2097.5 & 1423.9 & 8220.6 & 2625.8 & 142.8 & 3.1 & 2555.8 & 1804.0 & 10929.0 & 5565.0 \\
\hline PI_fixed & 100.5 & 2.9 & 2318.2 & 1593.1 & 11177.0 & 3955.8 & 170.0 & 3.5 & 2795.6 & 2675.4 & 15568.0 & 9641.1 \\
\hline PI_fixed_FTC & 91.8 & 2.6 & 2112.1 & 1425.2 & 8231.9 & 2611.6 & 141.0 & 3.2 & 2591.0 & 1819.1 & 11007.0 & 5737.0 \\
\hline PI_total & 151.6 & 2.7 & 2211.1 & 4272.6 & 17529 & 11496.0 & 235.5 & 3.4 & 2743.6 & 5097.3 & 21719.0 & 16887.0 \\
\hline PI_total_FTC & 91.8 & 2.6 & 2112.8 & 1425.7 & 8243.6 & 2628.1 & 141.0 & 3.1 & 2592.2 & 1819.7 & 11031.0 & 5686.4 \\
\hline PI_multi & 98.2 & 2.6 & 2154.2 & 1786.4 & 9382.7 & 3856.4 & 152.5 & 3.2 & 2263.5 & 2645.5 & 12817.0 & 6803.5 \\
\hline PI_multi_FTC & 91.3 & 2.6 & 2102.0 & 1415.3 & 8160.6 & 2657.8 & 140.6 & 3.1 & 2574.5 & 1802.7 & 10892.0 & 5655.5 \\
\hline LQR_bias & 76.9 & 3.0 & 2389.8 & 1835.4 & 10284.0 & 3139.5 & 115.9 & 3.6 & 2855.4 & 2401.8 & 11350.0 & 5166.5 \\
\hline LQR_bias_FTC & 55.8 & 2.9 & 2316.5 & 1549.9 & 7087.7 & 2669.1 & 97.2 & 3.5 & 2756.0 & 1933.7 & 8382.3 & 4359.0 \\
\hline LQR_fixed & 68.5 & 3.2 & 2522.5 & 1695.3 & 8005.0 & 3386.2 & 133.1 & 3.5 & 2841.9 & 2796.2 & 11029.0 & 6446.4 \\
\hline LQR_fixed_FTC & 55.5 & 2.9 & 2348.6 & 1559.2 & 7007.8 & 2677.6 & 97.1 & 3.5 & 2782.1 & 1928.7 & 8240.4 & 4932.7 \\
\hline LQR_total & 73.2 & 2.9 & 2325.8 & 4254.2 & 11981.0 & 5375.0 & 126.2 & 3.5 & 2830.2 & 5060.8 & 14116.0 & 7061.5 \\
\hline LQR_total_FTC & 55.7 & 2.9 & 2352.9 & 1556.3 & 6996.5 & 2736.0 & 96.7 & 3.5 & 2794.1 & 1928.4 & 8308.2 & 4944.6 \\
\hline LQR_multi & 60.1 & 2.9 & 2279.8 & 1709.9 & 7982.7 & 3425.5 & 101.8 & 3.5 & 2749.4 & 2534.4 & 9779.2 & 5586.0 \\
\hline LQR_multi_FTC & 55.1 & 2.9 & 2323.6 & 1544.4 & 6938.2 & 2687.0 & 96.8 & 3.5 & 2764.7 & 1922.9 & 8337.1 & 4391.0 \\
\hline
\end{tabular}




\section{References}

1. Lu Q, Bowyer R, Jones BL. Analysis and design of Coleman transform-based individual pitch controllers for wind-turbine load reduction. Wind Energy. 2015; 18(8): 1451-1468.

2. Malcolm DJ, Hansen AC. WindPACT Turbine Rotor Design Study: June 2000-June 2002 (Revised). tech. rep., National Renewable Energy Lab.(NREL); 2006.

3. Bossanyi EA, Fleming PA, Wright AD. Validation of individual pitch control by field tests on two-and three-bladed wind turbines. IEEE Transactions on Control Systems Technology. 2013; 21(4): 1067-1078.

4. Lio $\mathrm{WH}$, Jones BL, Rossiter JA. Estimation and control of wind turbine tower vibrations based on individual blade-pitch strategies. IEEE Transactions on Control Systems Technology 2018; 27(4): 1820-1828.

5. Leithead W, Dominguez S, Spruce C. Analysis of tower/blade interaction in the cancellation of the tower fore-aft mode via control. European Wind Energy Conference. 2004. London, Nov.

6. Darrow J, Johnson K, Wright A. Design of a tower and drive train damping controller for the three-bladed controls advanced research turbine operating in design-driving load cases. Wind Energy. 2011; 14(4): 571-601.

7. Plumley C, Leithead W, Jamieson P, Bossanyi E, Graham M. Comparison of individual pitch and smart rotor control strategies for load reduction. Journal of Physics: Conference Series 2014; 524(1): 012054.

8. Lio W, Jones BL, Rossiter J. Preview predictive control layer design based upon known wind turbine blade-pitch controllers. Wind Energy 2017; 20(7): 1207-1226.

9. Chilali M, Gahinet P. H/sub/spl infin//design with pole placement constraints: an LMI approach. IEEE Transactions on automatic control. 1996; 41(3): 358-367.

10. Shan M, Jacobsen J, Adelt S. Field testing and practical aspects of load reducing pitch control systems for a $5 \mathrm{MW}$ offshore wind turbine. In: ; 2013: 101-105.

11. Cho S, Gao Z, Moan T. Model-based fault detection of blade pitch system in floating wind turbines. Journal of Physics: Conference Series. 2016; 753(9): 092012.

12. Badihi H, Zhang Y. Fault-tolerant individual pitch control of a wind turbine with actuator faults. IFAC-PapersOnLine 2018; 51(24): 1133-1140.

13. Cho S, Gao Z, Moan T. Model-based fault detection, fault isolation and fault-tolerant control of a blade pitch system in floating wind turbines. Renewable energy 2018; 120: 306-321.

14. Shi F, Patton R. An active fault tolerant control approach to an offshore wind turbine model. Renewable Energy. 2015; 75: 788-798.

15. Chen L, Shi F, Patton R. Active FTC for hydraulic pitch system for an offshore wind turbine. 2013 IEEE Conference on Control and Fault-Tolerant Systems (SysTol). 2013: 510-515. Nice, Oct.

16. Rahnavard M, Ayati M, Yazdi MRH. Robust actuator and sensor fault reconstruction of wind turbine using modified sliding mode observer. Transactions of the Institute of Measurement and Control. 2019; 41(6): 1504-1518.

17. Wang L, Cai M, Zhang H, Alsaadi F, Chen L. Active fault-tolerant control for wind turbine with simultaneous actuator and sensor faults. Complexity 2017; 2017.

18. Odgaard PF, Stoustrup J, Kinnaert M. Fault-tolerant control of wind turbines: A benchmark model. IEEE Transactions on Control Systems Technology. 2013; 21(4): 1168-1182.

19. Jonkman J, Butterfield S, Musial W, Scott G. Definition of a 5-MW reference wind turbine for offshore system development. National Renewable Energy Laboratory, Technical Report No. NREL/TP-500-38060. 2009.

20. Duckwitz D, Shan M. Active tower damping and pitch balancing - design, simulation and field test. Journal of Physics: Conference Series. 2014; 555(1): 012030. 
21. Fischer B, Shan M. A survey on control methods for the mitigation of tower loads. Fraunhofer-Institute for Wind Energy and Energy Systems Technology, IWES Project report 2013; 1: 104-56.

22. Zhang Z, Nielsen SR, Blaabjerg F, Zhou D. Dynamics and control of lateral tower vibrations in offshore wind turbines by means of active generator torque. Energies. 2014; 7(11): 7746-7772.

23. Lio WH, Jones BL, Lu Q, Rossiter JA. Fundamental performance similarities between individual pitch control strategies for wind turbines. International Journal of Control 2017; 90(1): 37-52.

24. Bossanyi E. Further load reductions with individual pitch control. Wind Energy. 2005; 8(4): 481-485.

25. Wright A, Fingersh L. Advanced control design for wind turbines. Part I: Control Design, Implementation, and Initial Tests, National Renewable Energy Lab., USA. 2008.

26. Chaaban R, Ginsberg D, Fritzen CP. Structural Load Analysis of Floating Wind Turbines Under Blade Pitch System Faults: 301-334; Cham: Springer International Publishing . 2014

27. Liu Y, Patton RJ, Lan J. Fault-tolerant Individual Pitch Control using Adaptive Sliding Mode Observer. IFAC Safeprocess. 2018; 51(24): 11271132. Warsaw, Aug.

28. Laino DJ, Hansen AC. Aerodyn user's guide. available electronically at http://wind/designcodes/simulators/aerodyn/AeroDyn. pdf 2002.

29. Jonkman JM, Buhl Jr ML, others. FAST user's guide. National Renewable Energy Laboratory, Technical Report No. NREL/EL-500-38230. 2005.

30. Chen J, Patton RJ, Zhang HY. Design of unknown input observers and robust fault detection filters. International Journal of control. 1996; 63(1): 85-105.

31. Lan J, Patton RJ. A new strategy for integration of fault estimation within fault-tolerant control. Automatica 2016; 69: 48-59.

32. Naidu DS. Optimal control systems. CRC press . 2002.

33. Jonkman BJ. TurbSim user's guide: Version 1.50. National Renewable Energy Laboratory, Technical Report No. NREL/TP-500-46198. 2009.

34. Boyd S, El Ghaoui L, Feron E, Balakrishnan V. Linear matrix inequalities in system and control theory. volume 15. Siam . 1994. 